\title{
Antigen presentation by type 3 innate lymphoid cells instructs the differentiation of gut microbiota-specific regulatory $\mathrm{T}$ cells
}

Ranit Kedmi ${ }^{1}$, Tariq Najar ${ }^{1}$, Kailin R. Mesa ${ }^{1}$, Allyssa Grayson ${ }^{1,2}$, Lina Kroehling ${ }^{1}$, Yuhan Hao ${ }^{3,4}$, Stephanie $\mathrm{HaO}^{5}$, Maria Pokrovskii ${ }^{1,10}, \mathrm{Mo} \mathrm{Xu}^{1,11}$, Jhimmy Talbot ${ }^{1,12}$, Jiaxi Wang ${ }^{6}$,

Mark S. Anderson ${ }^{6}$, James M. Gardner ${ }^{6}$, Terri M. Laufer ${ }^{7}$, Iannis Aifantis ${ }^{8}$, Juliet M. Bartleson ${ }^{9,13}$, Paul M. Allen ${ }^{9}$, Marlon Stoeckius ${ }^{5,14}$, Dan R. Littman ${ }^{1,2 \dagger}$

1 Molecular Pathogenesis Program, The Kimmel Center for Biology and Medicine of the Skirball Institute, New York University School of Medicine, New York, NY, USA

2 Howard Hughes Medical Institute, New York, NY, USA

3 Center for Genomics and Systems Biology, New York University, New York, NY, USA

4 New York Genome Center, New York, NY, USA

5 Technology Innovation Lab, New York Genome Center, New York, NY, USA

6 Diabetes Center, University of California, San Francisco, San Francisco, CA, USA

7 Department of Medicine, Perelman School of Medicine, University of Pennsylvania and

Department of Medicine, C. Michael Crescenz Veterans Administration Medical Center, Philadelphia, PA, USA

8 Department of Pathology, New York University School of Medicine, New York, NY, USA

9 Department of Pathology and Immunology, Washington University School of Medicine, St.

Louis, MO, USA

Current addresses: ${ }^{10}$ Calico Life Sciences, LLC, South San Francisco, CA, USA; ${ }^{11}$ National Institute for Biological Sciences, Beijing, China; ${ }^{12}$ Fred Hutchinson Cancer Center, Seattle, WA USA; ${ }^{13}$ Buck Institute for Research on Aging, Novato, CA, USA $;{ }^{14} 10 X$ Genomics, Stockholm, Sweden

† E-mail: dan.littman@med.nyu.edu 


\section{Abstract}

The mutualistic relationship of gut-resident microbiota and cells of the host immune system promotes homeostasis that ensures maintenance of the microbial community and of a poised, but largely non-aggressive, immune cell compartment ${ }^{1,2}$. Consequences of disturbing this balance, by environmental or genetic factors, include proximal inflammatory conditions, like Crohn's disease, and systemic illnesses, both metabolic and autoimmune. One of the means by which this equilibrium is achieved is through induction of both effector and suppressor or regulatory arms of the adaptive immune system. In mice, Helicobacter species induce regulatory (iTreg) and follicular helper (Tfh) T cells in the colon-draining mesenteric lymph nodes under homeostatic conditions, but can instead induce inflammatory Th17 cells and colitis when iTreg cells are compromised $^{3,4}$. How Helicobacter hepaticus and other gut bacteria direct $\mathrm{T}$ cells to adopt distinct functions remains poorly understood. Here, we investigated which cells and molecular components are required to convey the microbial instruction for the iTreg differentiation program. We found that antigen presentation by cells expressing ROR $\gamma \mathrm{t}$, rather than by classical dendritic cells, was both required and sufficient for iTreg induction. These ROR $\gamma \mathrm{t}^{+}$cells, likely to be type 3 innate lymphoid cells (ILC3), require the MHC class II antigen presentation machinery, the chemokine receptor CCR7, and $\alpha_{v}$ integrin, which activates TGF- $\beta$, for iTreg cell differentiation. In the absence of any of these, instead of iTreg cells there was expansion of microbiota-specific pathogenic Th17 cells, which were induced by other antigen presenting cells (APCs) that did not require CCR7. Thus, intestinal commensal microbes and their products target multiple APCs with pre-determined features suited to directing appropriate T cell differentiation programs, rather than a common APC that they endow with appropriate functions. Our results illustrate the ability of microbiota to exploit specialized functions of distinct innate immune system cells, targeting them to achieve the desired composition of equipoised T cells, thus maintaining tolerance. 


\section{Main}

A subset of bacterial species among the hundreds that comprise the gut microbiota elicit stereotypic antigen-specific $\mathrm{T}$ cell differentiation programs through mechanisms yet to be elucidated. It is generally accepted that conventional (or classical) dendritic cells (cDC) that migrate from tissue to the inductive sites in lymph nodes present microbial antigens to activate and promote differentiation of naive antigen-specific $T$ cells $s^{4-9}$. However, the $\mathrm{cDC}$ that execute these functions according to instructions of distinct intestinal bacterial species have not been clearly defined. We have chosen to study APC requirements for T cell responses to $H$. hepaticus (Hh), which elicits iTreg, Tfh, or pathogenic Th17 cells under different conditions ${ }^{3}$.

\section{Antigen presentation by $R O R \gamma t^{+}$cells is required for microbiota-induced iTreg cell differentiation}

To study the properties of antigen-presenting cells that direct the differentiation of microbiotaspecific iTreg cells, we transferred into Hh-colonized mice naive Hh-specific $\mathrm{CD}^{+}{ }^{+} \mathrm{T}$ cells from HH7-2 TCR transgenic mice ${ }^{3}$. CFSE-labeled transferred T cells exhibited robust proliferation by day 3 in colon-draining $\mathrm{C} 1$ mesenteric lymph nodes (MLN) of wild type (WT) mice, with upregulation of Foxp3 and ROR $\gamma$ t, characteristic of colonic iTreg cells ${ }^{10,11}$. In contrast, in mice deficient for antigen presentation by DC (and, potentially, other cells: CD11c-Cre;/-A $b^{f / f}$, designated as $M H C I^{\Delta C D 11 c}$ ), there was no expression of Foxp3 by the Hh-specific T cells, but, surprisingly, there was substantial proliferation of these cells, with up-regulation of ROR $\gamma \mathrm{t}$ (Fig. 1a). At 2-3 weeks after transfer, there was massive expansion in the colonic lamina propria of ROR $\gamma$ t- and T-bet-expressing Hh-specific $\mathrm{T}$ cells in mutant mice, characteristic of a proinflammatory program (Extended Data Fig. 1a-c). Endogenous $\mathrm{T}$ cells also displayed this 
phenotype, with fewer Foxp3 ${ }^{+}$ROR $\gamma \mathrm{t}^{+}$iTreg and expansion of Th17 cells (Extended Data Fig. 1b).

This result suggested that antigen presentation by $\mathrm{CD}_{11 \mathrm{c}^{+}}$cells is required for iTreg cell differentiation, but that it is dispensable for the differentiation of the pro-inflammatory Th17 cells.

Classical DC, which have been broadly divided into $\mathrm{CDC} 1$ and $\mathrm{CDC} 2$, comprise multiple cell subsets that differ in their ontogeny, location, and transcription factor dependency ${ }^{12,13}$. Both $\mathrm{cDC} 1$ and $\mathrm{CDC} 2$ have been proposed to initiate iTreg responses, largely based on their ability to induce Treg cell differentiation in vitro ${ }^{8,14}$. However, in vivo depletion of $\mathrm{CDC} 1$ or $\mathrm{cDC} 2$ failed to phenocopy iTreg loss (Extended Data Fig. 1d-f), in agreement with previous reports ${ }^{5,15-17}$. These findings suggested that antigen presentation by a rare uncharacterized CD11 $\mathrm{c}^{+}$myeloid or nonmyeloid cell subset is required for iTreg cell differentiation. To identify putative antigen presenting cell populations targeted by CD11c-cre, we performed CITE-seq analysis of cells isolated from MLN of Hh-colonized CD11c-Cre;ROSA26LSLtdTomato (designated tdTomato-ON ${ }^{C D 11 c}$ ) fatemap mice. We identified both myeloid cell subsets and ILC3 that expressed MHC class II among

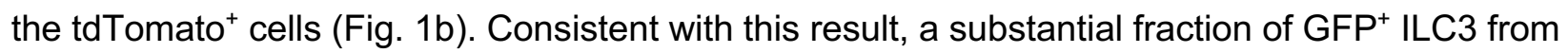
the MLN of ROR 1 -eGFP mice expressed CD11c and CD11b (Fig. 1c). These cells also expressed CD4 and CCR6, as well as high amounts of MHCII, markers of lymphoid tissue inducer (LTi)-like ILC3 (Fig. 1d). Flow cytometry analysis of cells from MLN of tdTomato-ON ${ }^{C D 11 c}$ fate-map mice showed elevated tdTomato reporter expression in the CD11 $\mathrm{b}^{+} \mathrm{CD} 11 \mathrm{c}^{+}$ILC3, but not in cells lacking these markers, suggesting recent expression of Cre in a subset of these cells (Fig. 1e). Moreover, many fewer ILC3 from MLN of $M H C / I^{\triangle C D 11 c}$ mice expressed $\mathrm{MHCII}$, as compared to WT littermates (Fig. 1f). Since CD11c and CD11b are considered to be CDC markers, we wanted to further bolster confidence as to their expression in ILC3. We therefore projected the CITE-seq data in FlowJo software ${ }^{18}$ and found that, in tdTomato-ON ${ }^{C D 11 c}$ mice, all tdTomato ${ }^{+}$ $\mathrm{CD}^{+}{ }^{+}$cells clustered as ILC3 or ILC1 (likely ex-ILC3), and not DC (Extended Data Fig. 1g). It 
was recently reported that a small subset of $\mathrm{cDC} 2$ express $\mathrm{ROR} \gamma \mathrm{t}$, and the study relied on discriminating ILC3 from CDC2 based on their expression of CD90 ${ }^{19}$. It should be noted that in our analysis a substantial fraction of $\mathrm{CD}^{-} 0^{-}$tdTomato $^{+}$cells from $t d$ Tomato-ON ${ }^{C D 11 c}$ mice also clustered as ILCs (Extended Data Fig. 1g,h), suggesting that CD90 might not be a faithful marker discriminating ILC3 from $\mathrm{CDC}$. This was substantiated by $R O R \not t$-Cre fate-mapping analysis of CD90 expression in tdTomato ${ }^{+}$cells isolated from the MLNs of $t d T_{\text {Tomato-ON }}{ }^{R O R_{\gamma t}}$ mice (Extended Data Fig. 1i). Thus, we conclude that the CD11 $\mathrm{C}^{+}$ROR $\gamma \mathrm{t}^{+}$cells most likely represent an ILC3 subset.

Expression of $\mathrm{MHCIl}$ by ILC3 was reported to prevent microbiota-dependent expansion of inflammatory $\mathrm{T}$ cells in the intestine, and was proposed to mediate negative selection of those cells $^{20}$. Our results suggested that CD11c-Cre-expressing ILC3 may, instead, be required for the differentiation of microbiota-specific iTreg cells. This was confirmed by examining iTreg cell differentiation in mice whose $\mathrm{MHCII}$ was inactivated in presumptive ILC3 (ROR $\gamma$-Cre;I-AB ${ }^{I /}$, designated as $\mathrm{MHCl}{ }^{\triangle R O R \gamma t}$ ). Despite expression of $\mathrm{MHCll}$ in $\mathrm{CDC} 2$ of these mutant mice (Fig. 2a), there was complete loss of Hh-specific iTreg cell differentiation in colon-draining MLN, but intact priming and subsequent expansion of pathogenic Th17 cells in the large intestine lamina propria, (Fig. 2b,c, Extended Data Fig. 2a). Both the donor Hh-specific and host endogenous T cells exhibited loss of iTreg and increase in IFN $\gamma$ - and IL-17A-producing CD4 ${ }^{+} \mathrm{T}$ cells in the large intestine lamina propria (Extended Data Fig. 2b-d). Similar results were observed with Hh-specific T cells in another conditional mutant strain, ROR $\nmid t-C r e ; H 2-D M a^{f / f}$ mice $\left(H 2-D M a^{\Delta R O R \nu t}\right)$ deficient in H2-DMa, the mouse equivalent of HLA-DM, required for displacement of invariant chain peptide and loading of processed peptide on MHCII molecules ${ }^{21}$. This result confirms that the antigen processing machinery is required in ILC3 for induction of microbiota-specific iTregs (Extended Data Fig. 2e). 


\section{Selective requirement for CCR7 expression in iTreg cell differentiation}

The chemokine receptor CCR7 mediates migration of DC and T cells into lymph nodes, where adaptive immune responses are initiated, and is critical for tolerogenic responses to food antigens $^{22}$. In CCR7-deficient mice, iTreg induction in response to $\mathrm{Hh}$ colonization failed (Extended Data Fig. 3a), in agreement with the recent demonstration of a CCR7 requirement for the differentiation of iTreg cells specific for other Helicobacter species ${ }^{5}$. However, we observed robust priming and proliferation of ROR $\gamma \mathrm{t}^{+} \mathrm{Hh}$-specific T cells in CCR7-deficient mice (Extended Data Fig. 3a). To determine which antigen presenting cells need to express CCR7 for iTreg cell differentiation, we examined mice with selective inactivation of Ccr7 in DCs and ILC $3^{23}$. In CD11CCre; $C \mathrm{cr} 7^{f / f}$ conditional mutant mice (designated $\operatorname{Ccr} 7^{\triangle C D 11 c}$ ), transferred Hh-specific T cells failed to differentiate into iTreg cells, despite exhibiting robust proliferation, as in CCR7-deficient mice (Fig. 3a). In the colon of these mice, Hh-specific iTreg cells were rare, but there was accumulation of inflammatory Th17 cells (Extended Data Fig. 3b,c) and appearance of tertiary lymphoid structures and elongated colonic crypts (Fig. 3b). Together, our results indicate that, unlike iTreg, microbiota-specific inflammatory Th17 cell differentiation does not depend on either CCR7 or antigen presentation by CD11c-expressing cells.

It was reported that CCR7 also mediates migration of intestinal ILC3 into draining MLN ${ }^{24,25}$. Notably, in the MLN of $C c r 7^{\triangle C D 11 c}$ mice, CD11 $\mathrm{b}^{+} \mathrm{CD} 11 \mathrm{c}^{+} \mathrm{ILC} 3$ lost CCR7 expression and were reduced in number (Extended Data Fig. 3d). Consistent with this observation, irradiated mice reconstituted with equal numbers of WT and $R O R \gamma t-C r e ; C C R 7^{f / f}\left(C c r 7^{\Delta R O R \gamma t}\right)$ bone marrow cells had fewer mutant than WT ILC3 in the C1 MLN. Importantly, the reduced population was among ILC3 that had the phenotype of lymphoid tissue inducer (LTi)-like cells (CCR6 $\left.{ }^{+}\right)$, while the NCR1+ ILC3 were slightly increased (Fig. 3c). However, there was no impact on (LTi)-like ILC3 counts in 
the gut, suggesting that CCR7 deficiency in ILC3 impacts their migration to the MLN, rather than development or survival (Fig. 3c). In line with this observation, Hh-specific iTreg cell differentiation was abrogated in the MLN of $C c r 7^{\Delta R O R y t}$ mice (Fig. 3d). Similarly, in the colon of $\operatorname{Ccr} 7^{\Delta R O R y t}$ mutant mice, there was expansion of Hh-specific T cells with the Th1-Th17 inflammatory program after 2-3 weeks of adoptive $\mathrm{T}$ cell transfer as was observed in $\mathrm{C} c r 7^{\Delta C D 11 c}$ mice (Fig. 3e). These results suggest that CD11C ${ }^{+}$ILC3 migrate to the MLN, where they present microbial antigen to naïve T cells to induce iTreg cell differentiation, and that their failure to migrate results, instead, in inflammatory Th17 cell differentiation and expansion.

\section{Integrin $\alpha_{v}$ expression by $\mathrm{ROR} \mathrm{t}^{+}$antigen presenting cells is required for iTreg differentiation}

Differentiation of iTreg cells requires TGF- $\beta$ signaling in $\mathrm{CD}^{+}{ }^{+} \mathrm{T}$ cells, and defects in this pathway result in spontaneous colitis ${ }^{3,10}$. TGF- $\beta$ is released from its latent form on cell surfaces or extracellular matrix following physical interaction with integrins $\alpha_{v} \beta_{6}$ or $\alpha_{v} \beta_{8}{ }^{26-28}$. Loss of integrins $\beta_{8}$ or $\alpha_{v}$ in hematopoietic cells, including in CD11c-Cre:Itgb8 $8^{f / f}$ mice, resulted in reduced colonic Tregs and in multiorgan inflammation ${ }^{29-31}$. Consistent with those observations, differentiation of adoptively transferred Hh-specific T cells into iTreg cells was abrogated both following treatment of mice with anti- $\alpha_{v} \beta_{8}$ antibody and in $C D 11 c-C r e$; Itgav $v^{f / f}\left(\right.$ Itgav $\left.^{\Delta C D 11 c}\right)$ recipient mice (Extended Data Fig. 4a).

Interestingly, in both CITE-seq and flow cytometry analyses of MLN cells, integrin $\alpha_{v}$ mRNA and cell surface protein were most highly expressed by ILC3 (Fig. 4a-c). Therefore we examined iTreg cell differentiation in mice with conditional inactivation of Itgav in presumptive ILC3 and in T cells $\left(\right.$ Itgav $\left.{ }^{\triangle R O R v t}\right)$ (Fig. 4c,d). In the colon-draining MLN of Itgav ${ }^{\triangle R O R \gamma}$ mice, adoptively transferred Hh- 
specific T cells failed to express Foxp3 (Fig. 4d). The MLN were increased in size (Extended Data

Fig. 4c) and the Hh-specific T cells expressed ROR $\gamma$ t, but, unlike in control littermates, they also had elevated T-bet, along with a substantial decrease in CCR6, consistent with reduced TGF- $\beta$ signaling (Extended Data Fig. 4d). Notably, $\alpha_{v} \beta_{8}$ antibody blockade resulted in the same phenotype (Extended Data Fig. 4e). In the colonic lamina propria of Itgav ${ }^{\Delta R O R \gamma t}$ mice, there was loss of both Hh-specific and host-derived iTreg cells, with skewing of CD4 ${ }^{+} \mathrm{T}$ cells towards IFN $\gamma^{+}$ Th1 and pathogenic Th17 programs (Fig. 4e, Extended Data Fig. 4f), suggesting that expression of integrin $\alpha_{v}$ on ILC3 is a general requirement for intestinal iTreg cell differentiation. Differentiation of iTreg cells was normal in CD4-Cre;/tgav ${ }^{f / f}$ mice (Extended Data Fig. 4g), consistent with a role of the integrin in ILC3 rather than in TCR $\alpha \beta$ T cells. Furthermore, we reconstituted mice after irradiation with a mix of $\mathrm{MHCII}^{\triangle C D 11 c}$ and Itgav ${ }^{\triangle R O R Y t}$ bone marrow cells, resulting in binary expression of $\mathrm{MHCll}$ or integrin $\alpha_{v}$. In these mice, iTreg cell differentiation was abolished, consistent with a requirement for both antigen presentation and activation of TGF- $\beta$ by the same ILC3, coupling T cell activation with differentiation cues (Fig. 4f,g, Extended Data Fig. 4h).

\section{Antigen presentation by ILC3 is sufficient to promote iTreg cell differentiation}

Our results indicated that antigen presentation by RORyt ${ }^{+}$cells is required to instruct microbiotaspecific iTreg cell differentiation. Even though these cells express the myeloid lineage markers CD11b and CD11c, they have phenotypic markers of LTi-like ILC3, including CD4, CD25, CCR6, CD127, and c-Kit. Because a small population of T-bet-negative cDC2 was recently reported to express ROR $\gamma \mathrm{t}^{19}$, and distinct Aire- and ROR $\gamma \mathrm{t}$-coexpressing APCs were also described ${ }^{32,33}$, we further investigated the properties of the ROR $\gamma \mathrm{t}^{+}$APCs. We used the zbtb46-eGFP model in an attempt to zoom in on cDCs, but not other myeloid or diverse cells targeted by CD11c-Cre. After confirming that $z b t b 46-C r e ; C c r 7^{f /-}$ mice $\left(C C R 7^{\Delta z b t b 46}\right)$ were also unable to support microbiota- 
dependent iTreg cell differentiation (Extended Data Fig. 5a), we examined C1 mLN cells from zbtb46-eGFP ; RORyt-Cre;ROSA26LSLtdTomato (tdTomato-ON ${ }^{R O R \gamma t}$ ) mice. We performed CITEseq with sorted cells expressing one or both fluorescent reporters, gated to exclude B and T cells. Unexpectedly, all ILC3 from MLN expressed both fluorescent reporters, indicating that ILC3 express Zbtb46 (Extended Data Fig. 5b). The fate-map analysis showed two cell subsets marked by $R O R \nmid t$-Cre expression: ILC3 and Aire ${ }^{+}$cells (recently named Janus cells ${ }^{32}$ ).However, the iTreg response to Hh was unimpaired in either RORyt-cre;Aire ${ }^{f / f}$ mice or in bone marrow chimeric mice reconstituted with Aire-DTR cells and treated with diphtheria toxin, ruling out these cells as the relevant APCs for iTreg instruction (Extended Data Fig. 5c,d). We also identified a few migratory cDC2 among the tdTomato GFP $^{+}$cells in zbtb46-eGFP ; tdTomato-ON ${ }^{R O R \gamma t}$ mice, but these did not exhibit active Rorc expression (Extended Data Fig. 5e), nor integrin $\alpha_{v}$ mRNA or protein expression, suggesting that they are unlikely to be ROR $\gamma \mathrm{t}^{+} \mathrm{APC}$ s required to direct iTreg cell differentiation.

Our results support a role for ILC3 in in microbiota-specific iTreg differentiation, but do not rule out a requirement for additional antigen presenting cells. We performed three-dimensional intravital imaging studies to visualize interactions of newly-primed Hh-specific T cells with DC and ILC3 populations in C1 MLN. Specifically, we generated mice expressing both zbtbCre;ROSA26LSLmKate2 (mKate2-ON ${ }^{z b t b 46}$ ) and ROR $\gamma$ t-eGFP, which differentially labeled cDC $\left(\mathrm{mKate}^{+}\right)$and ILC3 (eGFP ${ }^{+}$and mKate2). However, given that the efficiency of zbtb46-Cremediated activation of the mKate2 reporter was low ( $20 \%$ for ILC3 and CDC populations) (Extended Data Fig. 5f,g), we additionally utilized cell morphology and size analysis to distinguish the few host-derived GFP ${ }^{+}$T cells from GFP ${ }^{+}$ILC3. We next transferred dye-labeled Nur77-eGFP Hh-specific T cells into these fluorescent reporter host mice to measure direct interactions of 
primed T cells (which up-regulate Nur77) with DC and ILC3 at $15 \mathrm{~h}$ after adoptive transfer. Approximately $81 \%$ of $\mathrm{GFP}^{+}$primed Hh-specific T cells were found in contact with at least one ILC3 and/or DC, as opposed to only $\sim 31 \%$ of the non-primed T cells (Extended Data Fig. 6a,b).

Our imaging study and results with conditional mutant mice did not rule out a contribution by DC towards Hh-specific T cell activation and iTreg differentiation. We therefore wished to determine whether antigen presentation limited to only $R O R \gamma t$-Cre-expressing cells was sufficient to allow for iTreg cell differentiation. For this purpose, we used mice that express MHCIl only in presumptive ILC3, and not in DC or other APCs (ROR $\gamma t-C r e ; I-A B^{-/ I S I}$, designated MHCII-ON $\left.{ }^{R O R_{\gamma} t}\right)$. We reconstituted irradiated congenic mice with bone marrow from $M H C / I^{\triangle C D 11 c}$ mice with or without bone marrow from WT or $M H C I I-O N^{R O R \gamma t}$ mice (Fig. 5a). Flow cytometry analysis confirmed MHCII expression exclusively by ILC3 from the C1 MLN and the colon lamina propria of $\mathrm{MHCI}^{\mathrm{ACD11c}} ; \mathrm{MHClI-ON} \mathrm{NOR}, t^{2}$ bone marrow reconstituted mice (Fig. 5b, Extended Data Fig. 6a). As expected, in control mice reconstituted with only $\mathrm{MHCl}{ }^{\triangle C D 11 c}$ bone marrow cells, in which no $\mathrm{MHCll}$ expression was detected in either ILC3 or DC, there was no differentiation of adoptively transferred Hh-specific iTreg cells, but there was, instead, differentiation of ROR $\gamma \mathrm{t}^{+} \mathrm{Tbet}^{+}$ inflammatory Th17 cells (Fig. 5c,d). In contrast, antigen presentation by ROR $\gamma \mathrm{t}^{+}$cells alone was sufficient to rescue iTreg cell differentiation and suppression of inflammatory T cells in response

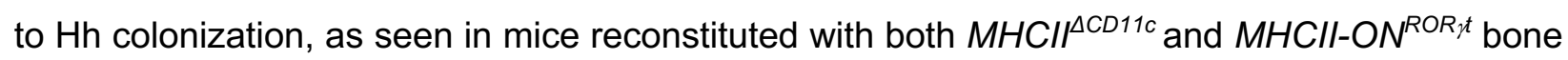
marrow cells (Fig. 5c,d). There was similar rescue of endogenous iTreg cells in mice having the gain-of-function MHCII in RORyt ${ }^{+}$cells (Extended Data Fig. 6b). It should be noted that whereas there was rescue of Hh-directed iTreg cell differentiation, there was a marked absence of Bcl6expressing Tfh cells, which are also induced by Hh (Fig. 5c,d). Interestingly, Tfh cells were present in $\mathrm{MHCII-ON}{ }^{C D 11 c}$ mice, suggesting that their differentiation requires antigen presentation by DC and/or B cells, as proposed previously ${ }^{34}$ (not shown). We conclude that ILC3 are specialized to 
prime naive microbiota-specific T cells and guide their differentiation into iTregs, but other APCs are required to guide the differentiation of microbiota-specific pathogenic Th17 cells and Tfh cells.

\section{Discussion}

The composition of the intestinal microbiota influences host immune functions that contribute to anti-microbial host defense, inflammatory disease, and anti-tumor immunity. Transmission of information from gut microbes to immune system cells remains poorly understood. The current results indicate that type 3 innate lymphoid cells, previously implicated in restraining microbiotadependent Th1/Th17 inflammatory responses in the gut, ${ }^{35,36}$ do so in large part by conveying signals from the microbiota to naive bacteria-specific $T$ cells, activating them and guiding their differentiation towards a unique iTreg cell program. ILC3 defective for CCR7-mediated migration, either to or within the MLN, MHCIl antigen presentation, or $\alpha v \beta 8$ function, presumably through activation of TGF- $\beta$, failed to induce the iTreg program. Intriguingly, under such circumstances there was expansion of pathogenic Th17 cells that promoted intestinal inflammation. We previously demonstrated that a T cell-intrinsic c-Maf deficiency prevented iTreg cell differentiation, and similarly allowed for microbiota-dependent differentiation of pathogenic Th17 cells ${ }^{3}$. Together, these findings suggest that iTreg cells restrain the priming, proliferation, and differentiation of Th17 cells in the MLN. The APC(s) that directs pathogenic Th17 cell differentiation does not require CCR7, and it is not targeted by using CD11c-Cre tools, and is, therefore, most likely not a $\mathrm{CDC}$, but its identity is currently not known. One hypothesis is that iTreg cells might not only inhibit Th17 cell differentiation and function but also may inhibit the function of the CCR7independent Th17 inducer APC. 
It has been proposed that intrinsically different APC subsets direct distinct T cell responses ${ }^{37}$, but such processes have been difficult to demonstrate in the setting of immune responses in vivo. Our study shows that a unique RORyt ${ }^{+}$cell type instructs naïve microbiota-specific $\mathrm{CD}^{+}{ }^{+} \mathrm{T}$ cells to become iTreg cells, but does not support the differentiation of other T cell programs, including Tfh cells, that are normally also induced by $\mathrm{Hh}$ intestinal colonization. Although our results are most compatible with the RORyt ${ }^{+}$APC being an ILC3 subset, most closely resembling the LTilike cells found in tertiary lymphoid tissues, we cannot rule out that it may represent a novel cell type that cannot yet be categorized as either lymphoid or DC-like. Definitive identification and characterization of this cell awaits more specific genetic tools than those currently available. Nevertheless, our results clearly demonstrate the existence of multiple APCs that are targeted by a specific commensal microbe to instruct diverse effector $T$ cell functions. The APCs may act hierarchically, as exemplified here by ILC3, which supersedes the function of the Th17-inducing APC. The existence and identity of distinct cellular circuits responsible for the induction of iTregs and other T cell functional subsets offers the opportunity to investigate the corresponding cells in humans and, potentially, to modulate them therapeutically.

\section{Acknowledgements}

We thank members of the Littman lab, Juan J. Lafaille, and Susan Schwab for valuable discussion and critical reading of the manuscript. We thank Dean Sheppard for providing ADWA11 blocking Ab, S.Y. Kim and the NYU Rodent Genetic Engineering Laboratory (RGEL) for rederivation of mutant mice, and Cindy Loomis and the Experimental Pathology Research Laboratory of NYULMC for histology of intestine samples. The Microscopy Core and the Genome Technology Core are partially supported by NYU Cancer Center Support Grant NIH/NCI P30CA016087 at the Laura and Isaac Perlmutter Cancer Center, S10 RR023704-01A1 and NIH S10 ODO01997401A1. The Experimental Pathology Research Laboratory is supported by National Institutes of 
Health Shared Instrumentation grants S100D010584-01A1 and S100D018338-01. This work was supported by an Irvington Institute fellowship from the Cancer Research Institute (R.K.) and a Jane Coffin Childs Fund fellowship (K.R.M.), the Helen and Martin Kimmel Center for Biology and Medicine (D.R.L.); National Institutes of Health grants R01Al139540 (P.M.A.) and R01Al158687 (D.R.L.), and the Howard Hughes Medical Institute (D.R.L.).

\section{Author Contributions}

R.K., T.N., K.R.M. and D.R.L. designed the study and analyzed the data; R.K. and T.N. performed the mouse genetic experiments with assistance from A.G; M.P., M.X., and J.T. performed early experiments to launch the study. Intravital multiphoton microscopy (K.R.M. and R.K.), CITE-seq studies (R.K, S.H. and M.S.), bioinformatics analyses (L.K. and Y.H.). J.W., M.S.A., and J.M.G. provided biological samples and experimental support. T.M.L., I.A., J.M.B., and P.M.A. contributed mouse strains. R.K. and D.R.L. wrote the manuscript, with input from the other authors. D.R.L. supervised the research.

\section{References}

1 Hooper, L. V., Littman, D. R. \& Macpherson, A. J. Interactions between the microbiota and the immune system. Science 336, 1268-1273, doi:10.1126/science.1223490 (2012).

2 Ansaldo, E., Farley, T. K. \& Belkaid, Y. Control of Immunity by the Microbiota. Annu Rev Immunol 39, 449-479, doi:10.1146/annurev-immunol-093019-112348 (2021).

$3 \mathrm{Xu}, \mathrm{M}$. et al. c-MAF-dependent regulatory T cells mediate immunological tolerance to a gut pathobiont. Nature 554, 373-377, doi:10.1038/nature25500 (2018). 
4 Chai, J. N. et al. Helicobacter species are potent drivers of colonic T cell responses in homeostasis and inflammation. Science immunology 2, doi:10.1126/sciimmunol.aal5068 (2017).

5 Russler-Germain, E. V. et al. Gut Helicobacter presentation by multiple dendritic cell subsets enables context-specific regulatory $T$ cell generation. eLife 10, doi:10.7554/eLife.54792 (2021).

6 Darrasse-Jèze, G. et al. Feedback control of regulatory T cell homeostasis by dendritic cells in vivo. The Journal of experimental medicine 206, 1853-1862, doi:10.1084/jem.20090746 (2009).

7 Durai, V. \& Murphy, K. M. Functions of Murine Dendritic Cells. Immunity 45, 719-736, doi:10.1016/j.immuni.2016.10.010 (2016).

8 Esterházy, D. et al. Classical dendritic cells are required for dietary antigen-mediated induction of peripheral T(reg) cells and tolerance. Nature immunology 17, 545-555, doi:10.1038/ni.3408 (2016).

9 Nussenzweig, M. C., Steinman, R. M., Gutchinov, B. \& Cohn, Z. A. Dendritic cells are accessory cells for the development of anti-trinitrophenyl cytotoxic $\mathrm{T}$ lymphocytes. The Journal of experimental medicine 152, 1070-1084, doi:10.1084/jem.152.4.1070 (1980).

10 Nutsch, K. et al. Rapid and Efficient Generation of Regulatory T Cells to Commensal Antigens in the Periphery. Cell Rep 17, 206-220, doi:10.1016/j.celrep.2016.08.092 (2016).

11 Esterhazy, D. et al. Compartmentalized gut lymph node drainage dictates adaptive immune responses. Nature 569, 126-130, doi:10.1038/s41586-019-1125-3 (2019).

12 Mildner, A. \& Jung, S. Development and function of dendritic cell subsets. Immunity 40, 642656, doi:10.1016/j.immuni.2014.04.016 (2014).

13 Anderson, D. A., 3rd, Dutertre, C. A., Ginhoux, F. \& Murphy, K. M. Genetic models of human and mouse dendritic cell development and function. Nat Rev Immunol 21, 101-115, doi:10.1038/s41577-020-00413-x (2021). 
14 Coombes, J. L. et al. A functionally specialized population of mucosal CD103+ DCs induces Foxp3+ regulatory $\mathrm{T}$ cells via a TGF-beta and retinoic acid-dependent mechanism. The Journal of experimental medicine 204, 1757-1764, doi:10.1084/jem.20070590 (2007).

15 Persson, E. K. et al. IRF4 transcription-factor-dependent CD103(+)CD11b(+) dendritic cells drive mucosal $\mathrm{T}$ helper 17 cell differentiation. Immunity 38, 958-969, doi:10.1016/j.immuni.2013.03.009 (2013).

16 Pool, L., Rivollier, A. \& Agace, W. W. Deletion of IRF4 in Dendritic Cells Leads to Delayed Onset of $\mathrm{T}$ Cell-Dependent Colitis. $J$ Immunol 204, 1047-1055, doi:10.4049/jimmunol.1900775 (2020).

17 Wohn, C. et al. Absence of MHC class II on cDC1 dendritic cells triggers fatal autoimmunity to a cross-presented self-antigen. Science immunology 5, doi:10.1126/sciimmunol.aba1896 (2020).

18 Hao, Y. et al. Integrated analysis of multimodal single-cell data. Cell 184, 3573-3587 e3529, doi:10.1016/j.cell.2021.04.048 (2021).

19 Brown, C. C. et al. Transcriptional Basis of Mouse and Human Dendritic Cell Heterogeneity. Cell 179, 846-863 e824, doi:10.1016/j.cell.2019.09.035 (2019).

20 Hepworth, M. R. et al. Immune tolerance. Group 3 innate lymphoid cells mediate intestinal selection of commensal bacteria-specific CD4(+) T cells. Science 348, 1031-1035, doi:10.1126/science.aaa4812 (2015).

21 Bartleson, J. M. et al. Strength of tonic T cell receptor signaling instructs $T$ follicular helper cell-fate decisions. Nature immunology 21, 1384-1396, doi:10.1038/s41590-020-0781-7 (2020).

22 Worbs, T. et al. Oral tolerance originates in the intestinal immune system and relies on antigen carriage by dendritic cells. The Journal of experimental medicine 203, 519-527, doi:10.1084/jem.20052016 (2006). 
23 Koscso, B. et al. Gut-resident CX3CR1(hi) macrophages induce tertiary lymphoid structures and IgA response in situ. Science immunology 5, doi:10.1126/sciimmunol.aax0062 (2020).

24 Mackley, E. C. et al. CCR7-dependent trafficking of RORgamma(+) ILCs creates a unique microenvironment within mucosal draining lymph nodes. Nat Commun 6, 5862, doi:10.1038/ncomms6862 (2015).

25 Kim, M. H., Taparowsky, E. J. \& Kim, C. H. Retinoic Acid Differentially Regulates the Migration of Innate Lymphoid Cell Subsets to the Gut. Immunity 43, 107-119, doi:10.1016/j.immuni.2015.06.009 (2015).

26 Wang, R. et al. GARP regulates the bioavailability and activation of TGFbeta. Mol Biol Cell 23, 1129-1139, doi:10.1091/mbc.E11-12-1018 (2012).

27 Lienart, S. et al. Structural basis of latent TGF-beta1 presentation and activation by GARP on human regulatory T cells. Science 362, 952-956, doi:10.1126/science.aau2909 (2018).

28 Qin, Y. et al. A Milieu Molecule for TGF-beta Required for Microglia Function in the Nervous System. Cell 174, 156-171 e116, doi:10.1016/j.cell.2018.05.027 (2018).

29 Lacy-Hulbert, A. et al. Ulcerative colitis and autoimmunity induced by loss of myeloid alphav integrins. Proc Natl Acad Sci U S A 104, 15823-15828, doi:10.1073/pnas.0707421104 (2007).

30 Paidassi, H. et al. Preferential expression of integrin alphavbeta8 promotes generation of regulatory T cells by mouse CD103+ dendritic cells. Gastroenterology 141, 1813-1820, doi:10.1053/j.gastro.2011.06.076 (2011).

31 Travis, M. A. et al. Loss of integrin alpha(v)beta8 on dendritic cells causes autoimmunity and colitis in mice. Nature 449, 361-365, doi:10.1038/nature06110 (2007).

32 Wang, J., Lareau, C.A., Bautista, J.L., Gupta, A.R., Sandor, K., Germino, J., Yin, Y., Arvedson, M.P., Reeder, G.C., Cramer, N.T., Xie, F., Ntranos,V., Satpathy, A.T., Anderson, M.S., Gardner, J.M. Single-cell multiomics defines tolerogenic extrathymic Aire-expressing populations with unique homology to thymic epithelium. Sci. Immunol. 6 (2021). 
33 Yamano, T. et al. Aire-expressing ILC3-like cells in the lymph node display potent APC features. The Journal of experimental medicine 216, 1027-1037, doi:10.1084/jem.20181430 (2019).

34 Barnett, L. G. et al. B cell antigen presentation in the initiation of follicular helper T cell and germinal center differentiation. J Immunol 192, 3607-3617, doi:10.4049/jimmunol.1301284 (2014).

35 Hepworth, M. R. et al. Innate lymphoid cells regulate CD4+ T-cell responses to intestinal commensal bacteria. Nature 498, 113-117, doi:10.1038/nature12240 (2013).

36 Goto, Y. et al. Segmented filamentous bacteria antigens presented by intestinal dendritic cells drive mucosal Th17 cell differentiation. Immunity 40, 594-607, doi:10.1016/j.immuni.2014.03.005 (2014).

37 Yin, X., Chen, S. \& Eisenbarth, S. C. Dendritic Cell Regulation of T Helper Cells. Annu Rev Immunol 39, 759-790, doi:10.1146/annurev-immunol-101819-025146 (2021). 
Figures

a
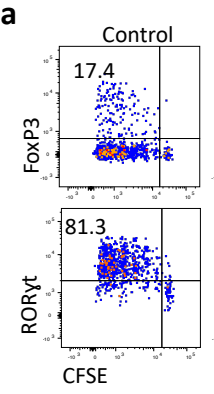

e

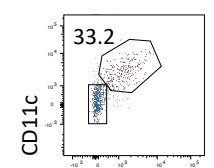

CD11b

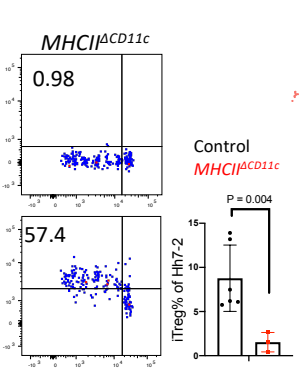

b
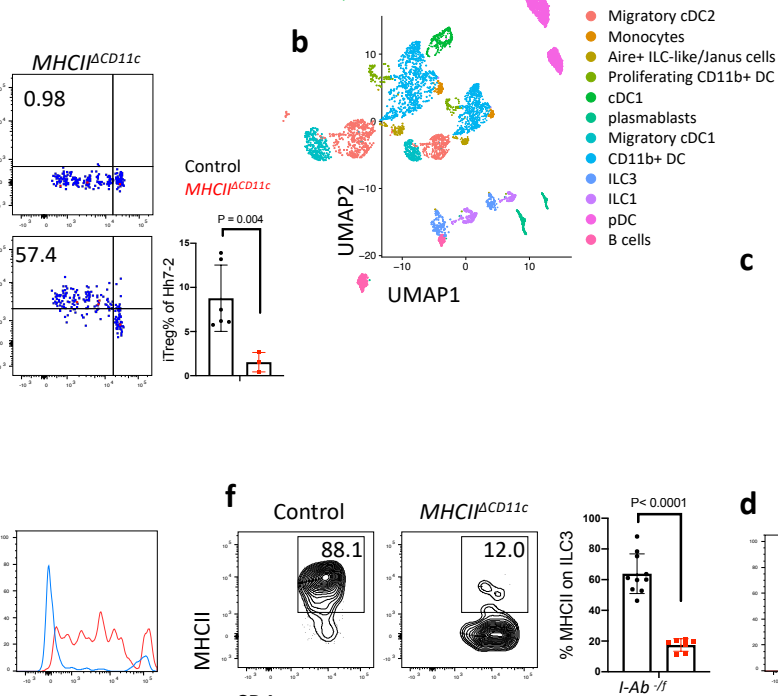

f

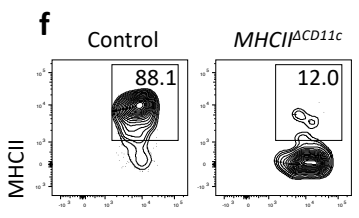

$\mathrm{CD} 4$

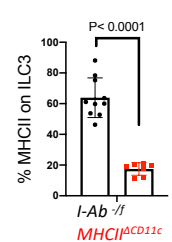

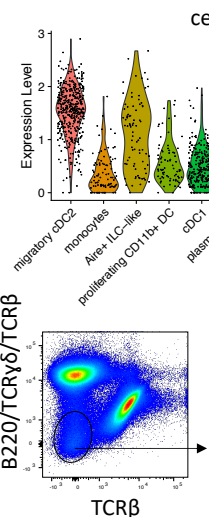

cell surface $\mathrm{MHClI}$

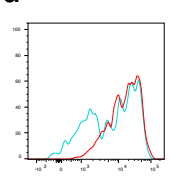

MHCII

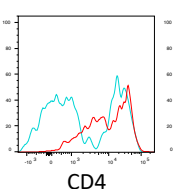

CD4
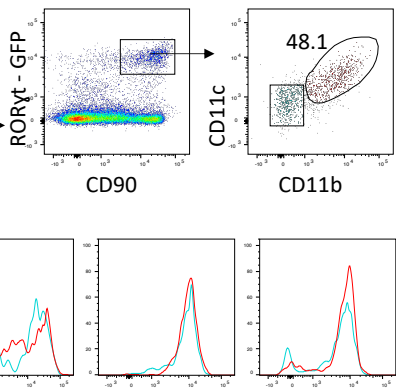

$\mathrm{CD} 25$

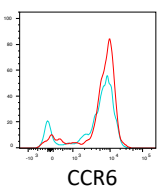

Figure 1. Expression of myeloid cell markers in ILC3.

a, Hh-specific T cell proliferation and differentiation in Hh-colonized $M H C / I^{\triangle C D 11 c}(n=3)$ and $I-A b^{f / f}$ or CD11c-Cre; I-A $b^{f /+}$ littermate control mice $(n=6)$. CFSE-labeled naïve TCR transgenic Hh7-2 T cells from the C1 MLN were assessed for cell proliferation and expression of FoxP3 and ROR $\gamma \mathrm{t}$ at 3 days after adoptive transfer. Representative flow cytometry (left) and aggregate results (right). Data summarize two independent experiments. b, UMAP visualization of the tdTomato-ON ${ }^{C D 11 c}$ fate-map cell CITE-seq dataset, analyzed by the WNN method (left), and Violin plot showing $\mathrm{MHCll}$ protein levels in the different cell clusters (right). MLN cells from $\mathrm{Hh}$-colonized tdTomato$O N^{C D 11 c}$ fate-map mice were gated for TCR $\beta^{-}, \mathrm{TCR}_{\gamma} \delta^{-}, \mathrm{B} 220^{-}$, and tdTomato ${ }^{+}$cells were sorted for CITE-seq analysis. Cells were sorted from two mice and labeled by hashing antibodies $(n=2)$. c, CD11C and CD11b staining of ILC3 from MLN of Hh-colonized mice, gated as indicated. d, Flow

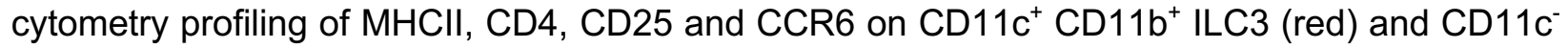
CD11b ILC3 (light blue) gates in (c). e, TdTomato levels in CD11 $\mathrm{c}^{+}$CD11 $\mathrm{b}^{+}$ILC3 (red) and CD11 $\mathrm{c}^{-}$CD11 $\mathrm{b}^{-}$ILC3 (blue) from the MLN of Hh-colonized tdTomato-ON ${ }^{C D 11 c}$ fate-map mice. $\mathbf{f}$, $\mathrm{MHCll}$ expression in ILC3 isolated from the C1 MLN of $\mathrm{MHCII}^{1 \mathrm{CD} 11 \mathrm{C}}(\mathrm{n}=7)$ and control littermates $(n=10)$. Data summarize three independent experiments. All statistics were calculated by unpaired two-sided Welch's t-test. Error bars denote mean \pm s.d. $p$-values are indicated in the figure. 

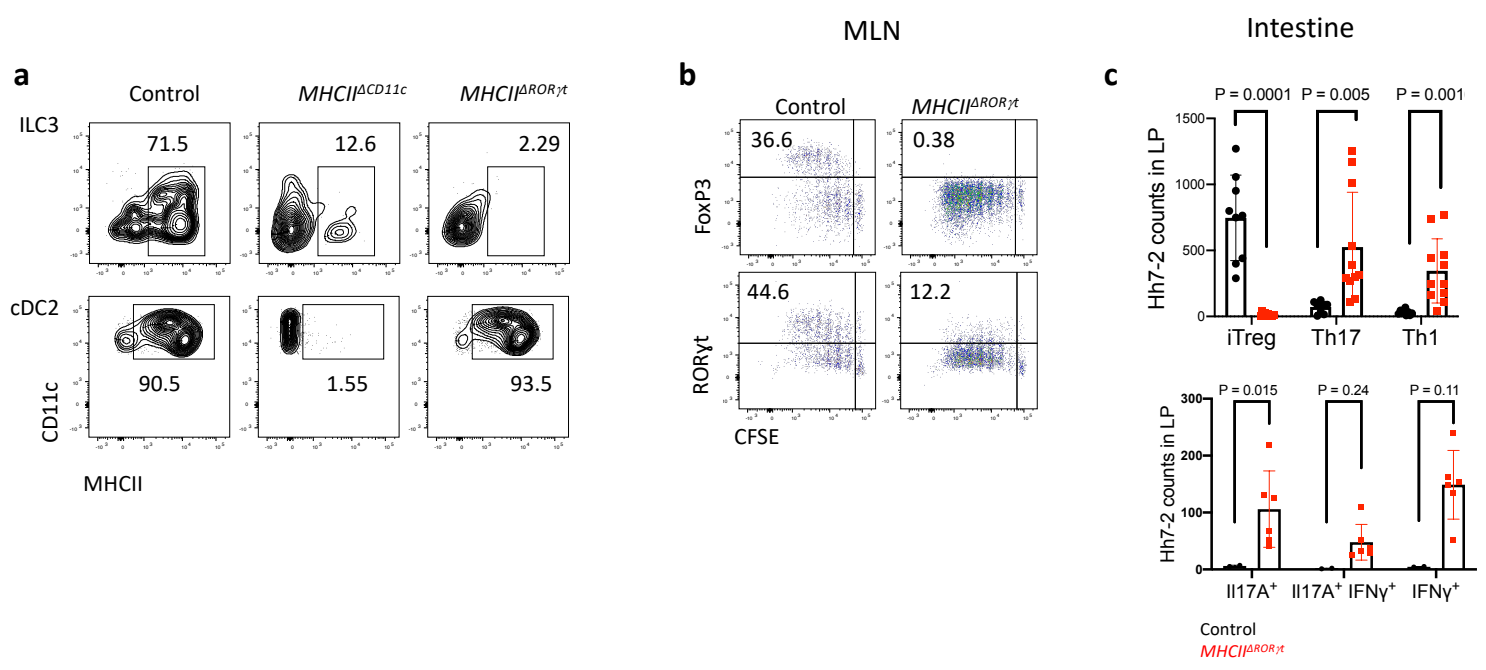

Figure 2. Antigen presentation by RORyt ${ }^{+}$cells is required for microbiota-induced iTreg cell differentiation. a, MHCIl expression in ILC3 (top) and cDC2 (bottom) from the MLN of Hhcolonized mice of the indicated genotypes. The ILC3 were gated as TCR $\beta$, TCR $\gamma \delta^{-}, \mathrm{B} 220^{-}$, $\mathrm{ROR} \mathrm{t}^{+}, \mathrm{CCR}^{+}, \mathrm{CD}^{+} 5^{+}$; DC were gated as TCR $\beta^{-}, \mathrm{TCR} \gamma \delta^{-}, \mathrm{B} 220^{-}, \mathrm{CD} 90^{-}, \mathrm{CD} 11 \mathrm{C}^{+}, \mathrm{CD} 11 \mathrm{~b}^{+}$ SIRPa $\alpha^{+} . \mathbf{b}, \mathrm{Hh} 7-2$ cell proliferation and differentiation in $M H C I^{1 R O R, t}(\mathrm{n}=6)$ and $I-A b^{f f f}$ littermate control mice $(n=6)$ at 3 days after adoptive transfer into Hh-colonized mice. c, Hh7-2 T cell differentiation profiles (upper) and cytokine production (lower) in the large intestine lamina propria at 22 days after transfer into $M H C I^{\perp R O R, t}(n=11)$ and littermate controls $(n=10)$. Differentiation was assessed by expression of Foxp3, RORyt with or without T-bet, and T-bet. All statistics were calculated by unpaired two-sided Welch's t-test. Error bars denote mean \pm s.d. $p$-values are indicated on the figure. Data summarize two independent experiments. 
a

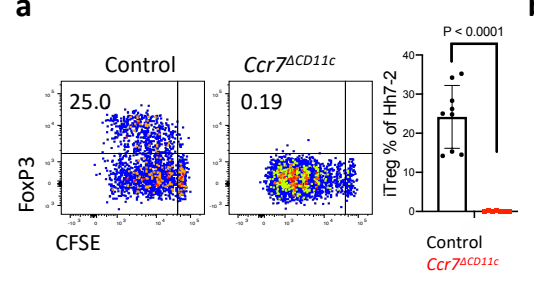

d

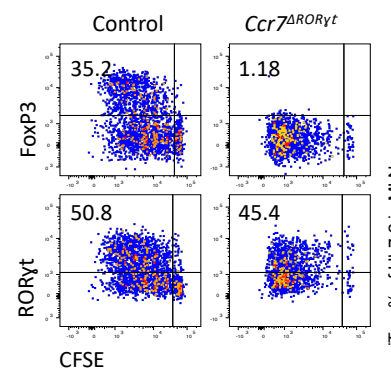

b
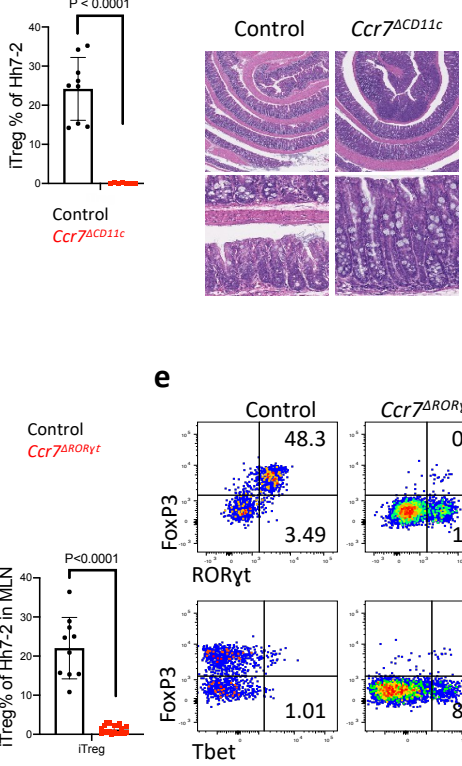

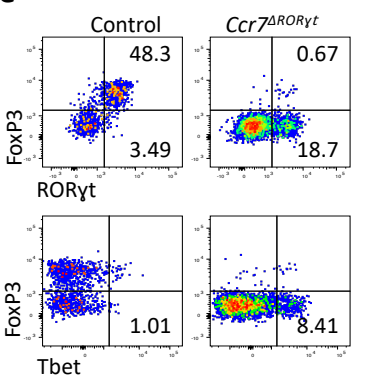

c
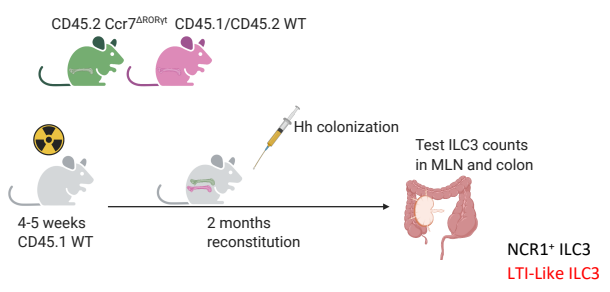

MLN

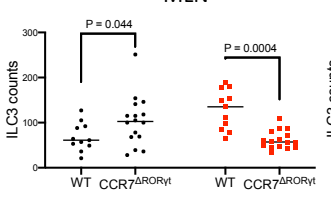

Colon LP

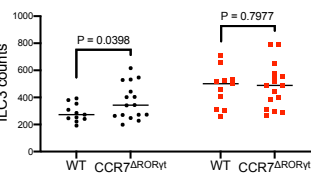

Figure 3. Requirement for innate immune cell CCR7 expression in differentiation of iTreg, but not pathogenic Th17 cells. a, Representative flow cytometry profiles (left) and aggregate data (right) for Hh7-2 T cell proliferation and differentiation in MLN at 3 days following adoptive transfer into $\operatorname{Ccr}^{\Delta \mathrm{CD11c}}(\mathrm{n}=7)$ and littermate control mice $(n=9)$. b, Representative H\&E histology in large intestine of mice with indicated genotypes. c, Number of ILC3 of each subset (NCR $1^{+}$and $\mathrm{CCR}^{+} / \mathrm{LTi}$-like) in colon lamina propria and MLN of mixed bone marrow chimeric mice reconstituted with cells from control and $\operatorname{Ccr} 7^{\triangle R O R \gamma t}$ mice. Scheme is shown at the top (created with BioRender.com). Irradiated CD45.1 mice were reconstituted with equal number of cells from CD45.2 Ccr7 $^{\triangle R O R y t}$ and CD45.1/CD45.2 WT mice or with CD45.2 WT and CD45.1/CD45.2 WT mice as controls. After 2 months mice were colonized with Hh. For statistical analysis, total counts of LTi-like and NCR1 ${ }^{+}$ILC3 counts were compared between CD45.2 WT and Ccr7 ${ }^{\triangle R O R y t}$ bone marrow-derived cells. d-e, Representative flow cytometry profiles (left) and aggregate data (right) for Hh7-2 T cell proliferation and differentiation in MLN at 3 days (d) and phenotype in large intestine at 14 days (e) following adoptive transfer into $C c r 7^{\triangle R O R \gamma t}$ and littermate control mice. MLN: Control mice $n=10, C c r 7^{\triangle R O R \gamma t}$ mice $n=20$; LI: Control mice $n=10, \operatorname{Ccr} 7^{\triangle R O R \gamma t}$ mice $n=9$. Data summarize three independent experiments. All statistics were calculated by unpaired two-sided Welch's t-test. Error bars denote mean \pm s.d. $p$-values are indicated in the figure. 

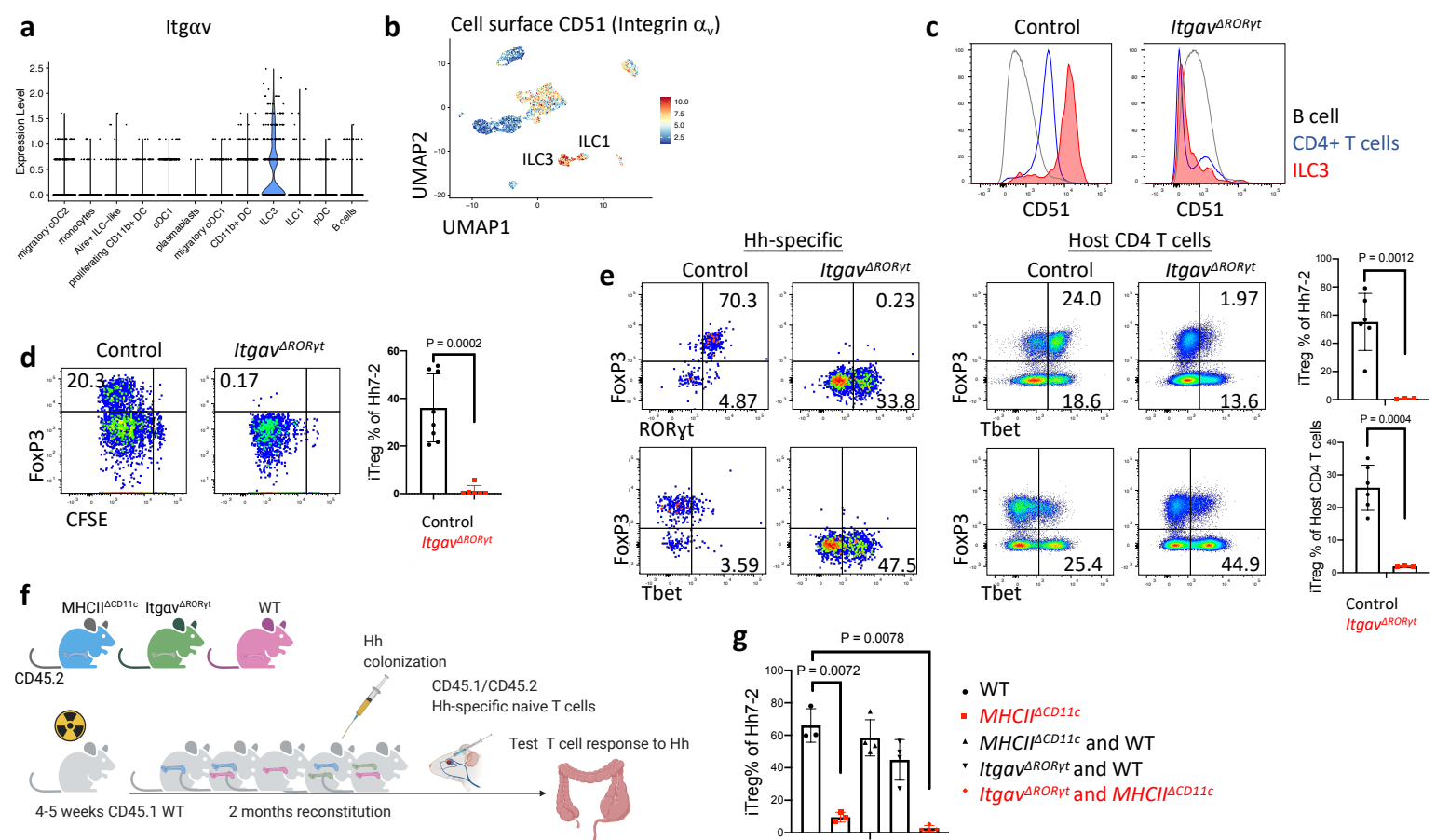
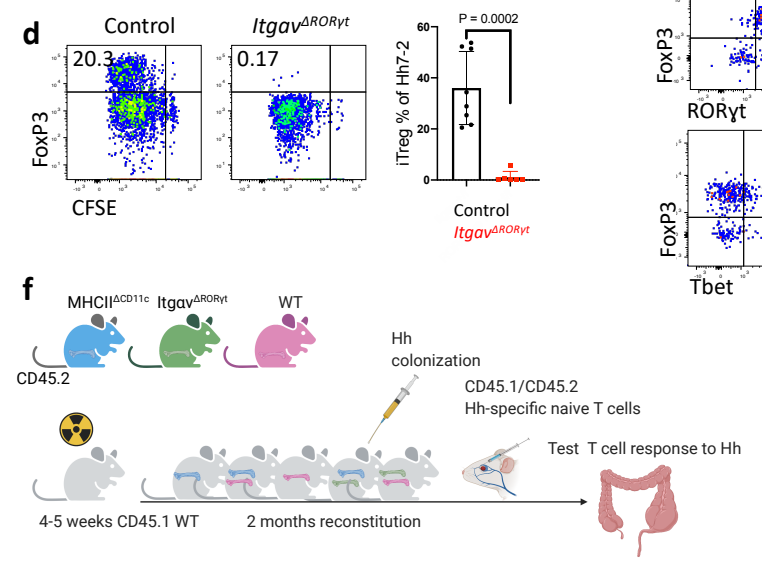

colonization

Test $\mathrm{T}$ cell response to $\mathrm{Hh}$

Figure 4. Role of integrin $\alpha_{v} \beta_{8}$ in $\mathrm{ROR}_{\gamma} \mathrm{t}^{+}$antigen presenting cell-dependent iTreg cell differentiation. $\mathbf{a}$, Violin plot of Itgav mRNA expression and $\mathbf{b}$, relative expression levels of cell surface CD51 (integrin $\alpha_{v}$ ) in CITE-seq-assigned clusters of sorted tdTomato-ON ${ }^{C D 11 c}$ fatemapped cells from the C1 MLN (described in Fig. 1b). c, Flow cytometry histograms showing CD51 levels on B cells, T cells and ILC3 from Itgav ${ }^{\triangle R O R y t}$ and littermate control mice. d, Proliferation and differentiation of Hh-specific iTreg cells in the MLN of Itgav ${ }^{\triangle R O R y t}(\mathrm{n}=6)$ and littermate control mice $(n=8)$. CFSE-labeled Hh7-2 T cells were analyzed at 3 days following their adoptive transfer into Hh-colonized mice. Representative flow cytometry profiles (left) and aggregate data (right). Data summarize three independent experiments. e, Transcription factor expression in Hh7-2 T cells (left panels) and in endogenous CD4 ${ }^{+} \mathrm{T}$ cells (right panels) from colon lamina propria (LP) at 10 days after adoptive transfer into Itgav ${ }^{\Delta R O R Y t}$ mice $(n=3)$ and control littermates $(n=6)$. Data summarize two independent experiments. Representative dot plots and aggregate data are shown in (d) and (e). f, Scheme for mixed bone marrow chimeric mouse experiment, with control, Itgav ${ }^{\Delta R O R Y t}$ or $M H C I^{\triangle C D 11 c}$ cells administered to irradiated host mice (created with BioRender.com). (g) Bar graphs showing iTreg frequency among Hh7-2 T cells in the colon LP at 10 days after their transfer into the bone marrow chimeric mice, reconstituted with different combinations of donor cells as indicated. Control mice $(n=3), M^{2} \|^{\triangle C D 11 c}(n=3)$, $\mathrm{MHCII}^{\triangle C D 11 c}$ and WT $(n=4)$, Itgav ${ }^{\triangle R O R Y t}$ and WT $(n=4)$ and $\mathrm{MHCII}^{\triangle C D 11 c}$ and Itgav ${ }^{\triangle R O R y t}(n=4)$. All statistics were calculated by unpaired two-sided Welch's t-test. Error bars denote mean \pm s.d. $p$ values are indicated in the figure. 
a

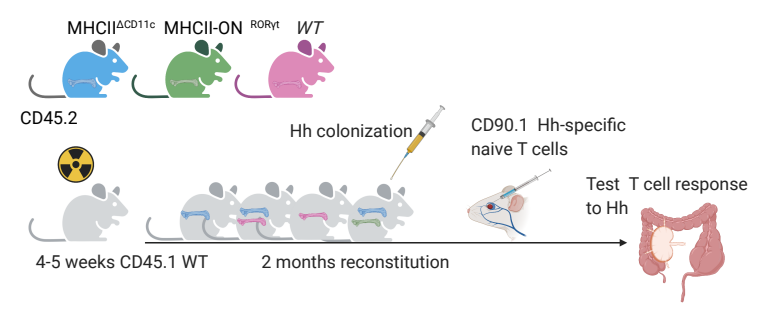

C WT MHCIIACD11C MHCIIACD11C MHCIIICD11C

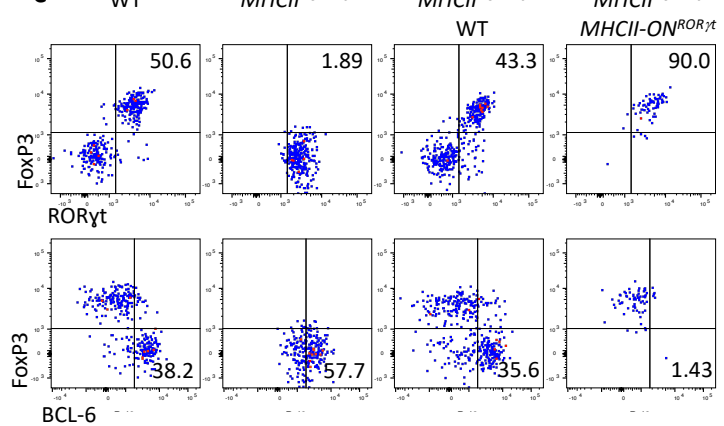

d b
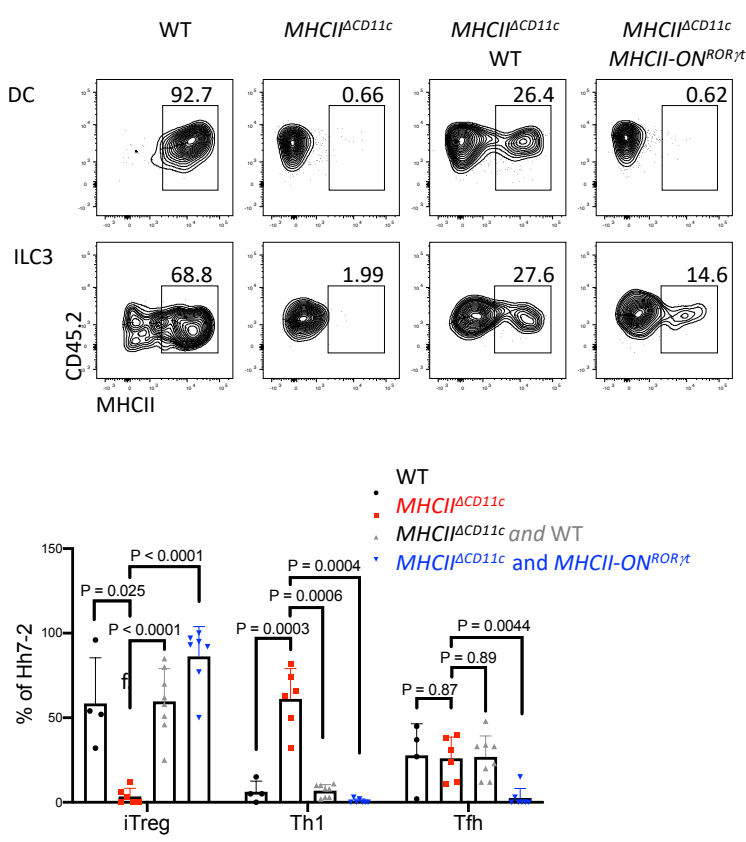

Figure 5. Antigen presentation by $R O R \gamma t^{+}$cells is sufficient to promote iTreg cell differentiation. a, Experimental design (created with BioRender.com). b, MHCII frequency in

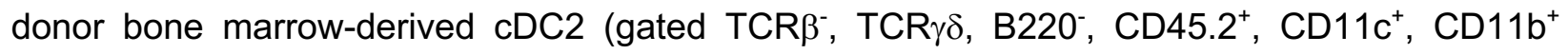
Sirpa $^{+}$) and ILC3 (gated as TCR $\beta^{-}, \mathrm{TCR}_{\gamma} \delta^{-}, \mathrm{B} 220^{-}, \mathrm{ROR}_{\mathrm{f}}{ }^{+}, \mathrm{CD}^{+}, \mathrm{CD} 45.2^{+}$) in MLN from chimeric mice reconstituted with combinations of donor BM cells as indicated. c, Representative flow cytometry of Hh7-2 T cell differentiation in colon lamina propria of Hh-colonized bone marrow chimeric mice, 12 days after transfer of naive TCR transgenic T cells. d, Aggregate data for differentiation of Hh7-2 T cells in bone marrow chimeric mice reconstituted with cells of indicated genotypes. WT (n=4), MHCII ${ }^{\triangle C D 11 c}(n=6), \mathrm{MHCl}^{\triangle C D 11 c}$ and WT $(n=8), \mathrm{MHCl}^{\triangle \mathrm{CD} 11 \mathrm{c}}$ and MHCII$\mathrm{ON}^{\Delta R O R y t}(n=7)$. All statistics were calculated by unpaired two-sided Welch's t-test. Error bars denote mean \pm s.d. $p$-values are indicated in the figure. 


\section{Extended Data}

a

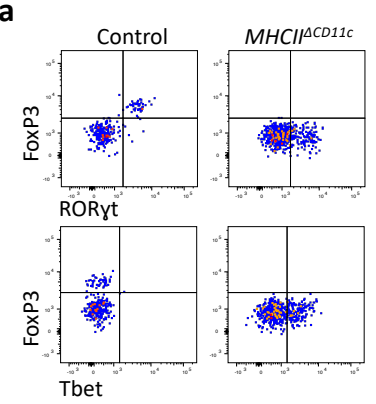

d $B A T F 3 \cdot /+$

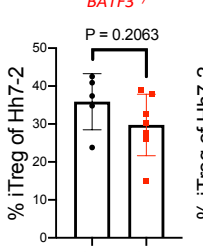

e $\operatorname{lif} 4 / 1+$

CD11c cre:irf4t $\stackrel{P=0.4328}{P}$

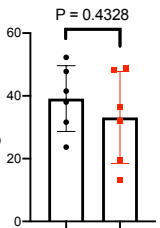

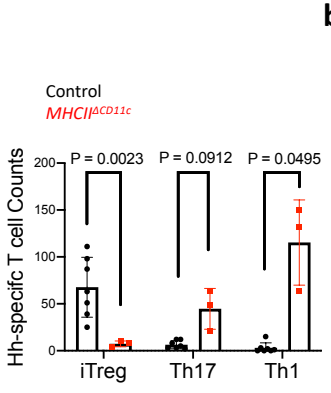

b

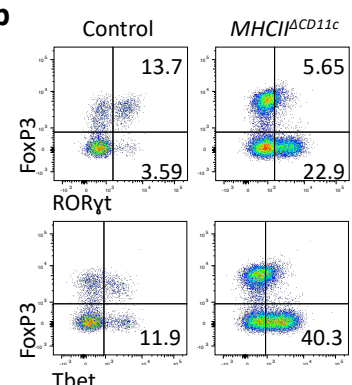

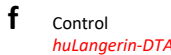

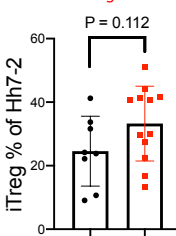

g

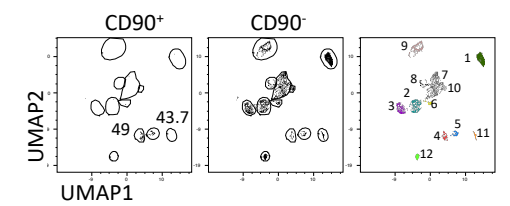

i

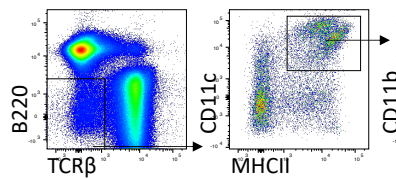

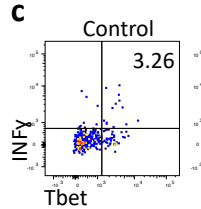
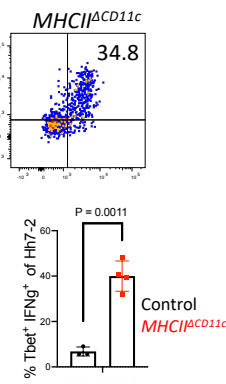

$\mathbf{h}$
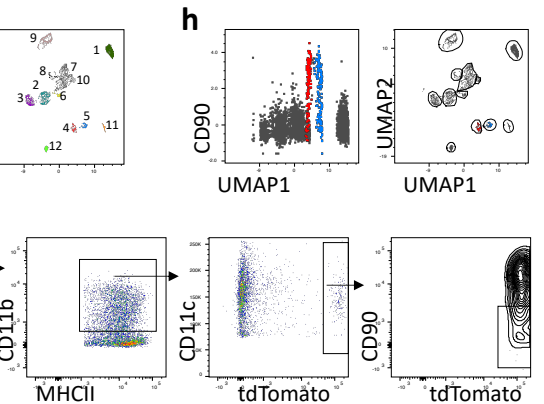

Extended Data Fig. 1. Cells targeted by CD11C-Cre and consequences for Hh-specific T cell differentiation. a, Phenotype of Hh7-2 TCR transgenic T cells in the colon lamina propria at 10 days after transfer into Hh-colonized $\mathrm{MHCI}^{\Delta C D 11 c}(\mathrm{n}=3)$ and control mice $(\mathrm{n}=7)$, as indicated. b, Phenotype of host CD4 ${ }^{+} \mathrm{T}$ cells from mice in (a). c, Cytokine profile of Hh7-2 T cells shown in (a). d-f, Proportion in MLN of Hh7-2 with the iTreg phenotype at 3 days after transfer into BATF3/. (d) ( $\mathrm{n}=7), \operatorname{IRF}^{\Delta C D 11 c}(\mathrm{e})(\mathrm{n}=6)$, and huLangerin (CD207)-DTA (f) ( $\left.\mathrm{n}=12\right)$ mice (red) and indicated littermate controls (black). Data summarize at least two independent experiments. g, FlowJo analysis of tdTomato-ON ${ }^{C D 11 c}$ fate map CITE-seq data (Fig. 1b) showing CD90 ${ }^{+}$and CD90 cells, presented in UMAP clustering coordinates (1: pDC, 2: migratory $\mathrm{CDC} 2,3$ : migratory $\mathrm{cDC} 1,4$ : ILC3, 5: ILC1, 6: Aire ${ }^{+}$ILC-like, 7: CD11b+ DC, 8: proliferating CD11b+ DC, 9: resident CDC1, 10: monocytes, 11: plasmablasts, 12: B cells). h, CD90 cell surface levels of tdTomato-ON ${ }^{C D 11 c}$ CITEseq data analyzed by FlowJo. i, Flow cytometry analysis of cells isolated from the C1 MLN of ROR $\gamma$ t fate-map mice ( $t d T o m a t o-O N^{R O R \gamma^{t}}$ ), as indicated. All statistics were calculated by unpaired two-sided Welch's t-test. Error bars denote mean \pm s.d. $p$-values are indicated in the figure. 
a

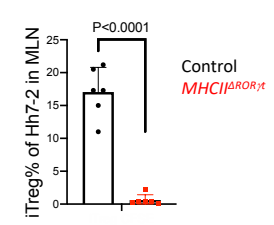

d
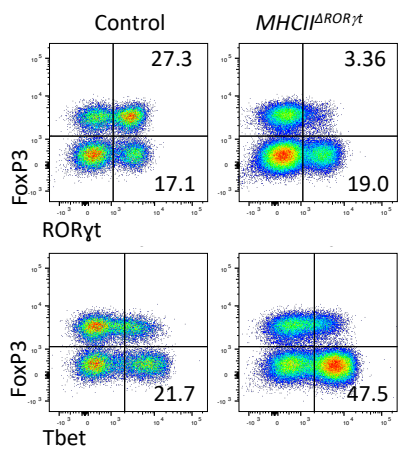
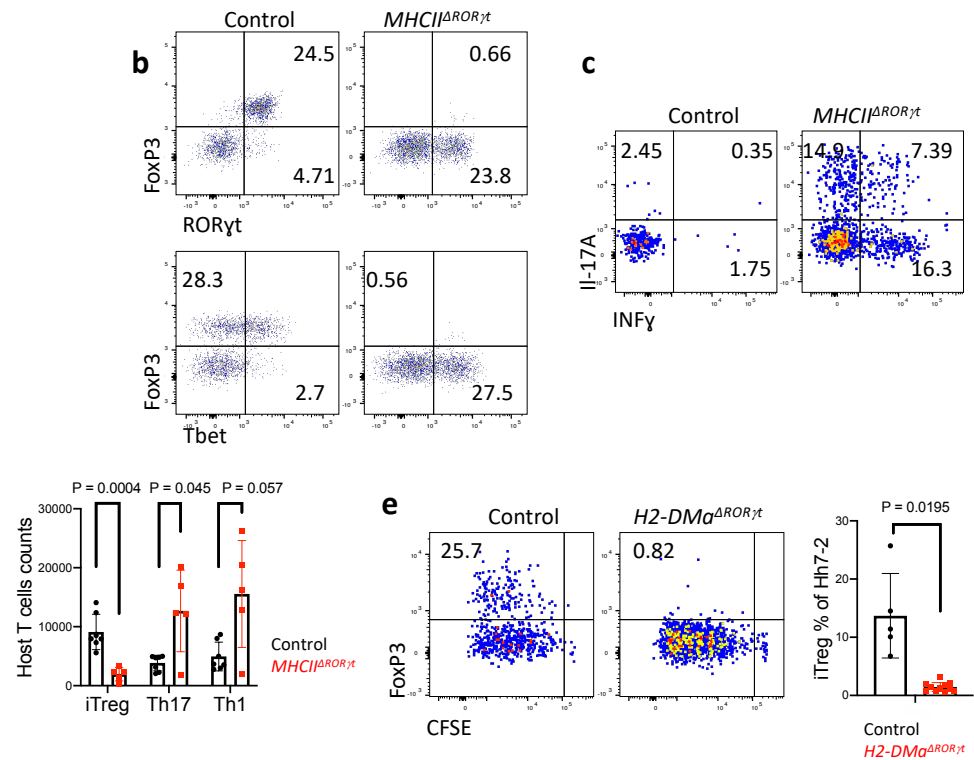

Extended Data Fig. 2. Antigen presentation by RORyt ${ }^{+}$cells is required for microbiotainduced iTreg cell differentiation. a, Bar graph showing frequency of iTreg among Hh7-2 T cells, measured as in Figure 2b. b-c, Representative dot plots showing Hh7-2 T cell differentiation (b) and cytokine (c) profiles in colon lamina propria at 22 days after adoptive transfer into Hhcolonized $M H C / I^{\perp R O R, t}$ and littermate controls. $\mathbf{d}$, Representative (left) and aggregate data of transcription factor profiles of host $\mathrm{CD} 4^{+} \mathrm{T}$ cells in colon lamina propria of mice shown in (b) and (c). e, Hh7-2 cell proliferation and differentiation in the MLN of H2-DMa ${ }^{\triangle R O R, t}$ (RORyt-Cre; H2$\left.\mathrm{Dma}^{f / f}\right)(\mathrm{n}=11)$ and littermate controls (RORyt-Cre; $\left.\mathrm{H2}-\mathrm{DMa}^{+/ f}\right)(\mathrm{n}=5)$ at 3 days after transfer of CFSE labeled naïve Hh7-2, cell proliferation and FoxP3 were assessed in cells isolated from C1 MLN. Representative flow cytometry (left) and aggregate data from multiple animals (right). Data summarize two independent experiments. All statistics were calculated by unpaired two-sided Welch's t-test. Error bars denote mean \pm s.d. $p$-values are indicated in the figure. 
a
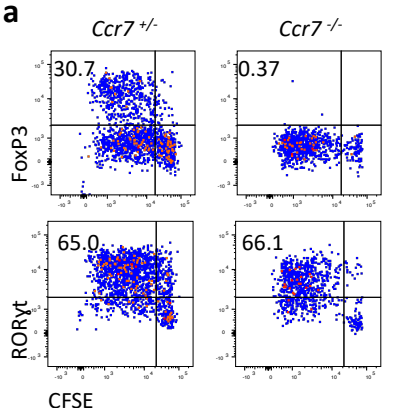

C

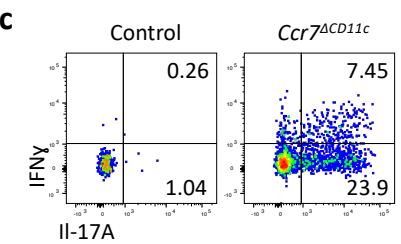

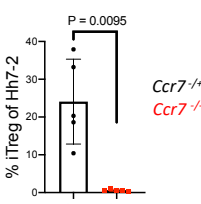

b
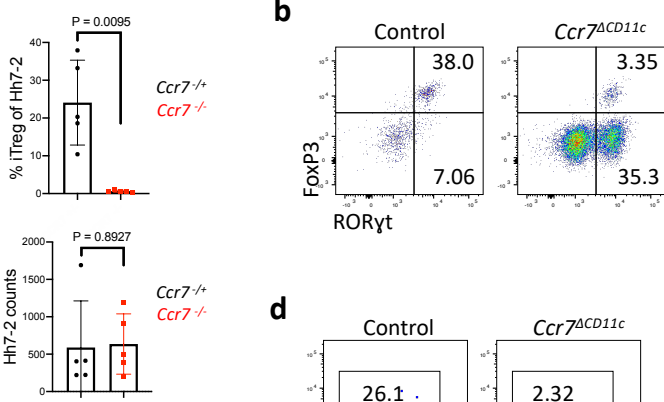

d

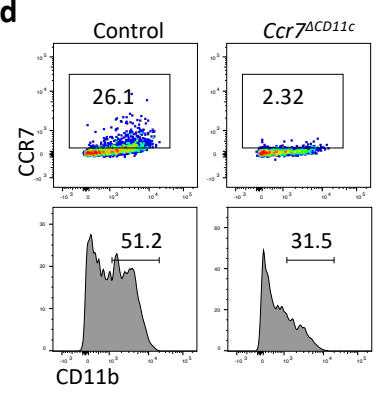

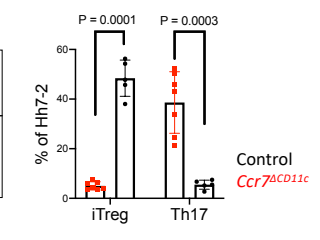

Extended Data Fig. 3. Differential requirements for CCR7 in iTreg and effector Th17 cell differentiation. a, Proliferation and differentiation of Hh-specific iTreg and Th17 cells in the MLN of $\mathrm{Ccr7}^{-1-}(n=5)$ and littermate control mice $(n=5)$. CFSE-labeled Hh7-2 T cells were analyzed at 3 days following their adoptive transfer into Hh-colonized mice. Representative flow cytometry profiles (left) and aggregate data (right). Data summarize two independent experiments. b-c, Transcription factor (b) and intracellular cytokine (c) profiles of Hh7-2 T cells in the large intestine of $\operatorname{Ccr}^{\Delta C D 11 c}(n=7)$ and littermate control $(n=5)$ mice, at 10 days after adoptive transfer. Aggregate data shown at the right in (b). Data summarize two independent experiments. d, Cell surface

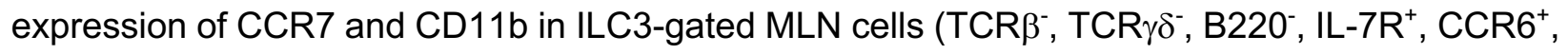
$C D 25^{+}$) from $C c r 7^{\triangle C D 11 c}$ and control Hh-colonized mice. All statistics were calculated by unpaired two-sided Welch's t-test. Error bars denote mean \pm s.d. $p$-values are indicated on the figure. 


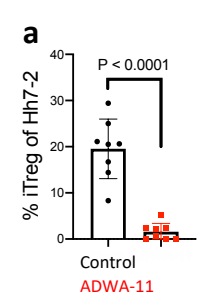

b
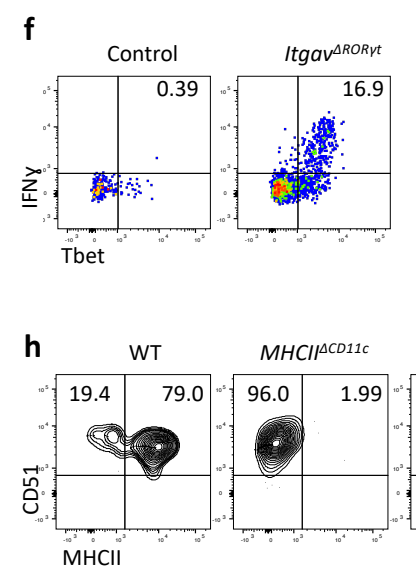

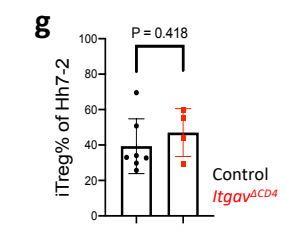

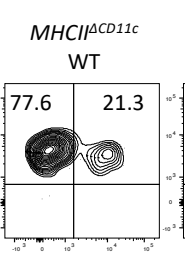

c
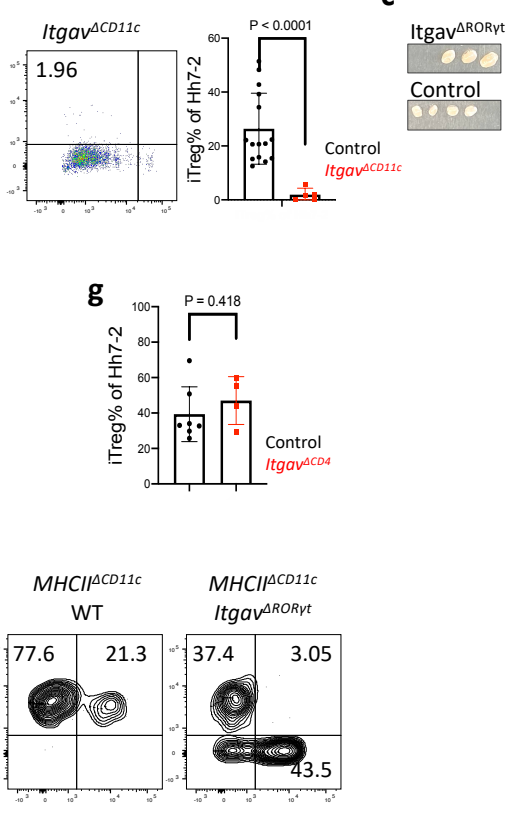

d
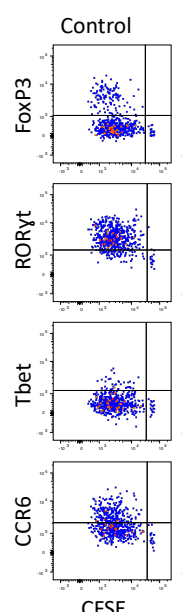

CFSE e
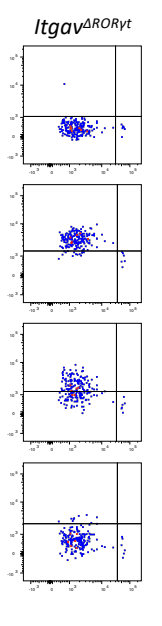
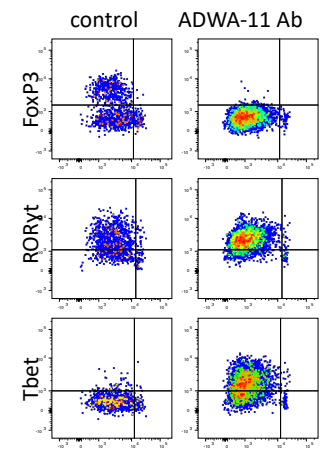

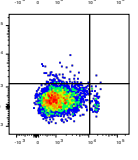

Extended Data Fig. 4. Effect of integrin $\alpha_{v} \beta_{8}$ blockade or $\alpha_{v}$ inactivation on microbiotadependent $\mathbf{T}$ cell differentiation. a, Frequency of iTreg cells among proliferating donor-derived Hh-specific cells in the MLN at 3 days after transfer of naïve CFSE-labeled Hh7-2 T cells into mice treated with $200 \mu \mathrm{g}$ of ADWA11 blocking antibody $(n=8)$ or left untreated $(n=8)$, on the day of adoptive transfer. Data summarize three independent experiments. b, Hh7-2 T cell proliferation and differentiation in the MLN of Itgav ${ }^{\triangle C D 11 c}(n=5)$ and littermate controls $(n=15)$ at 3 days after adoptive transfer. Data summarize three independent experiments. c, C1 MLN from Itgav ${ }^{\triangle C D 11 c}$ $(n=3)$ and littermate controls $(n=4), 10$ days after Hh colonization. d-e, Flow cytometry profiling of transcription factors and CCR6 in proliferating CFSE-labeled Hh7-2 in the MLN at 3 days after adoptive transfer into Itgav ${ }^{\triangle R O R y t}$ and littermate control mice (d) or into mice treated with ADWA11 (as in a) or untreated control littermates (e). f, Intracellular IFN $\gamma$ and T-bet expression in PMA/lonomycin-stimulated Hh7-2 T cells isolated from colon lamina propria of Itgav $^{\Delta R O R y t}$ and control littermates, 10 days after adoptive transfer. $\mathbf{g}$, Frequency of iTreg cells among proliferating Hh7-2 in the MLN at 3 days after adoptive transfer into Itgav ${ }^{\triangle C D 4}(\mathrm{n}=4)$ and control littermates $(n=7)$. Data summarize two independent experiments. $h$, CD51 (Integrin $\alpha_{v}$ ) and MHCIl cell surface expression in ILC3 (gated as TCR $\beta^{-}, \mathrm{TCR} \gamma \delta^{-}, \mathrm{B} 220^{-}, \mathrm{ROR} \gamma \mathrm{t}^{+}, \mathrm{CD}^{+}, \mathrm{CD} 25^{+} \mathrm{CD} 45.2^{+}$) isolated from MLN of bone marrow chimeric mice, reconstituted with different combinations of donor cells as indicated and colonized with $\mathrm{Hh}$ for 21 days. All Statistics were calculated by unpaired two-sided Welch's t-test. Error bars denote mean \pm s.d. $p$-values are indicated on the figure. 


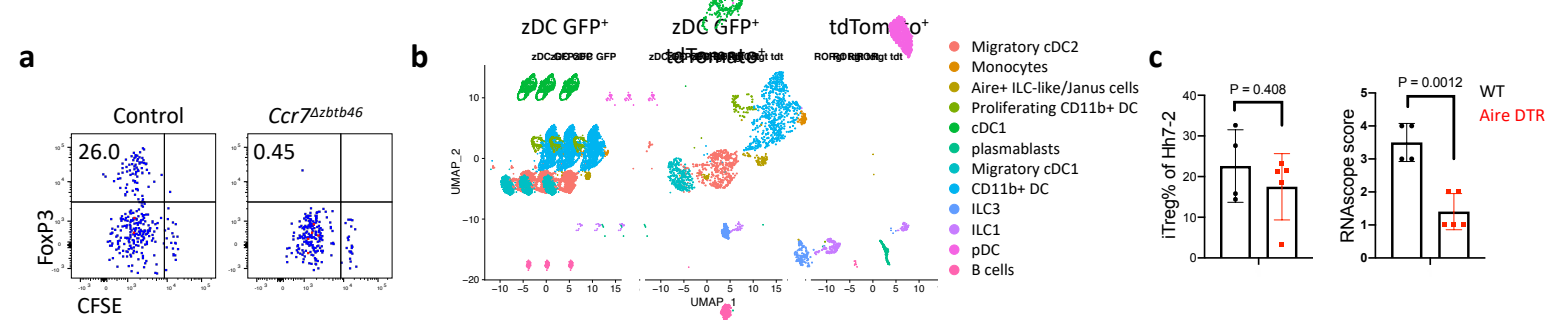

d

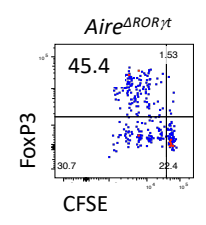

e

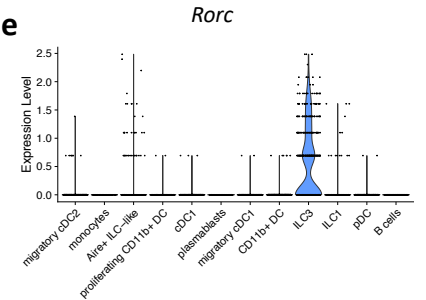

f

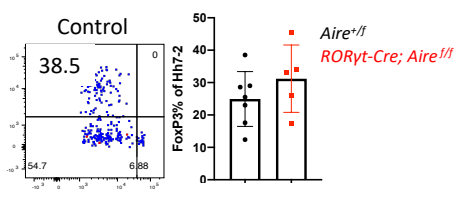

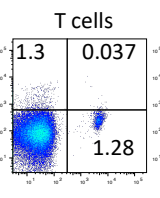
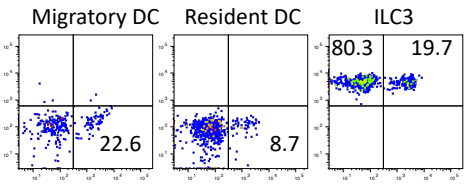

g
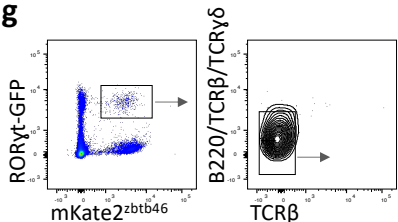
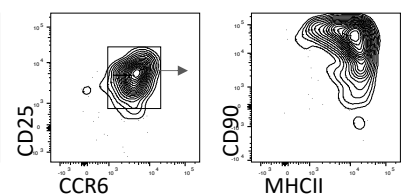

Extended Data Fig. 5. Analysis of ROR and differentiation in MLN of $C c r 7^{\Delta z b t b 46}$ and control littermates, at 3 days after transfer of the naïve cells. b, UMAP visualization of CITE-seq datasets obtained from 3 distinct sorted populations $\left(\mathrm{GFP}^{+}, \mathrm{GFP}^{+}\right.$tdTomato $^{+}$and tdTomato ${ }^{+}$) isolated from C1 MLN of Zbtb46-eGFP ; tdTomato$O N^{\triangle R O R \gamma t}$ mice $(\mathrm{n}=2)$, analyzed by the WNN method, . c, Bone marrow chimeric mice were reconstituted with BM cells from CD45.2 Aire-DTR or CD45.2 WT mice. One month after reconstitution, mice were colonized with $\mathrm{Hh}$, and one week later were treated with Diphtheria toxin (DT, Sigma-Aldrich) for 3 sequential days (at a dose of $25 \mathrm{ng} / \mathrm{g}$ mice). CD45.1 CD45.2 CFSE labeled Hh7-2 T cells $\left(1 \times 10^{5}\right)$ were transferred intravenously into the mice on the first day of DT treatment. Bar graph of proportion of proliferating Foxp3 ${ }^{+} \mathrm{Hh} 7-2 \mathrm{~T}$ cells in the MLN of mice reconstituted with Aire-DTR BM $(n=5)$ or with WT BM $(n=4)$ (left); Aire mRNA in the spleen of the treated mice was blindly scored using RNAscope analysis. d, Proliferation and differentiation of CFSE-labeled Hh7-2 T cells in the MLN of ROR $\gamma$-Cre;Aire ${ }^{f / f}(\mathrm{n}=5)$ and control Aire ${ }^{+/ f}$ littermates $(n=8)$ at 3 days after adoptive transfer. Data summarize three independent experiments. e, Violin plot showing Rorc levels in the cell clusters identified in the CITE-seq analysis in (b). f, Flow cytometry analysis of fate-mapped C1 MLN cells from ROR $\gamma$ t-eGFP;mKate2-ON ${ }^{\Delta z b t b 46}$ mice, gated for the indicated cell subsets. Note that there is incomplete excision of the transcriptional stop signal by zbtb46-Cre. g, Phenotypic analysis of eGFP ${ }^{+} \mathrm{mKate}^{+}$cells from the double reporter mice described in (e). All Statistics were calculated by unpaired two-sided Welch's t-test. Error bars denote mean \pm s.d. $p$-values are indicated on the figure. 
a
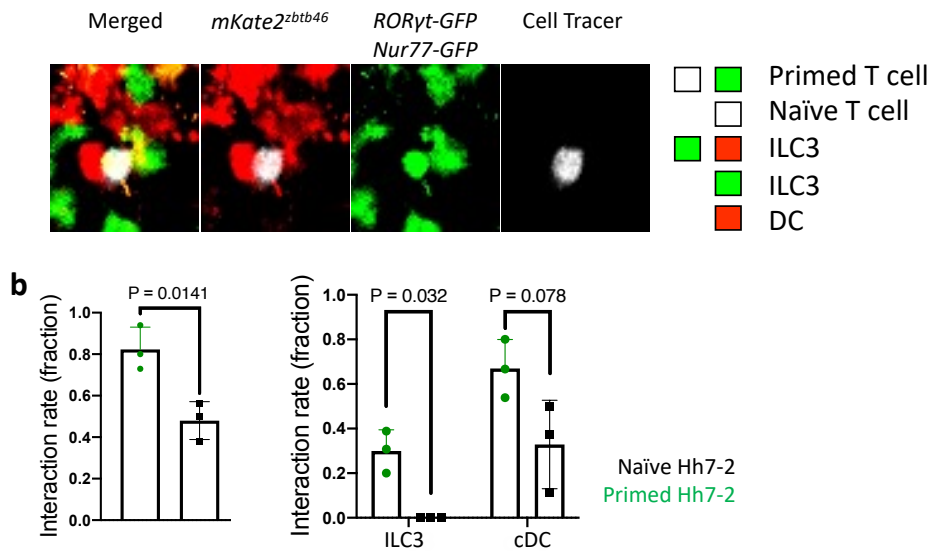

Extended Data Fig. 6. Intravital tracking of ILC3 and DC interactions with Hh-specific T cells during priming in the MLN. a, Representative image of cell-cell interactions of recently primed Hh-specific T cells with DC and ILC3. Nur77-eGFP tracer-labeled Hh7-2 T cells were transferred into of ROR $\nmid$-eGFP;mKate2-ON ${ }^{2 b t b 46} \mathrm{Hh}$-colonized mice. Cell colocalization of primed Hh7-2 (tracer dye ${ }^{+}, \mathrm{GFP}^{+}$) or naïve Hh7-2 (tracer dye ${ }^{+}$, GFP-) T cells with cDC (mKate2 ${ }^{+}$with dendritic morphology), ILC3 (eGFP ${ }^{+}, \mathrm{mKate}^{+}$or $\mathrm{eGFP}^{+}$alone with amoeboid morphology), or both were visualized using intravital multiphoton microscopy of the C1 MLN at $15 \mathrm{~h}$ after transfer. Note that Cell Tracer fluorescent labeling provides clear spatial discrimination of $R O R \not t-$ eGFP and Nur77-eGFP expressing cells. b, Quantification and graphical representation of the total and individual rates of interaction of ILC3 or CDC populations with primed or naïve Hh7-2 T cells. Data summarize cell-cell interactions from six $0.25 \mathrm{~mm}^{3}$ three-dimensional regions of $\mathrm{C} 1$ MLN, ( $n=72$ total Hh7-2 T cells), ( $n=49$ primed and 23 naïve Hh7-2 T cells). All statistics were calculated by unpaired two-sided Welch's t-test. Error bars denote mean \pm s.d. $p$-values are indicated in the figure. 


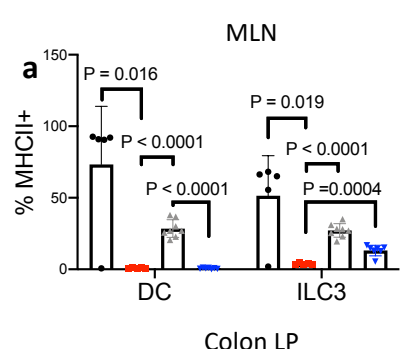

\section{b}
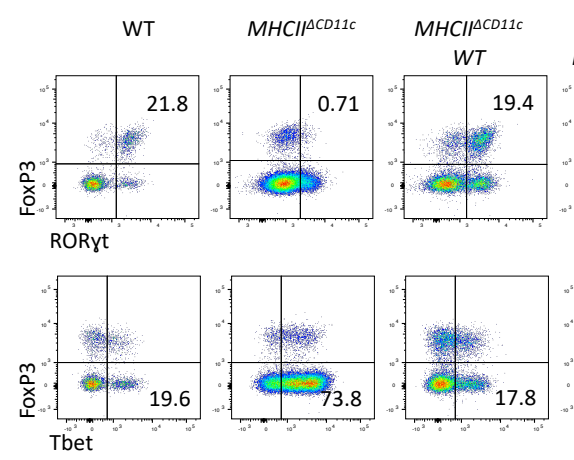
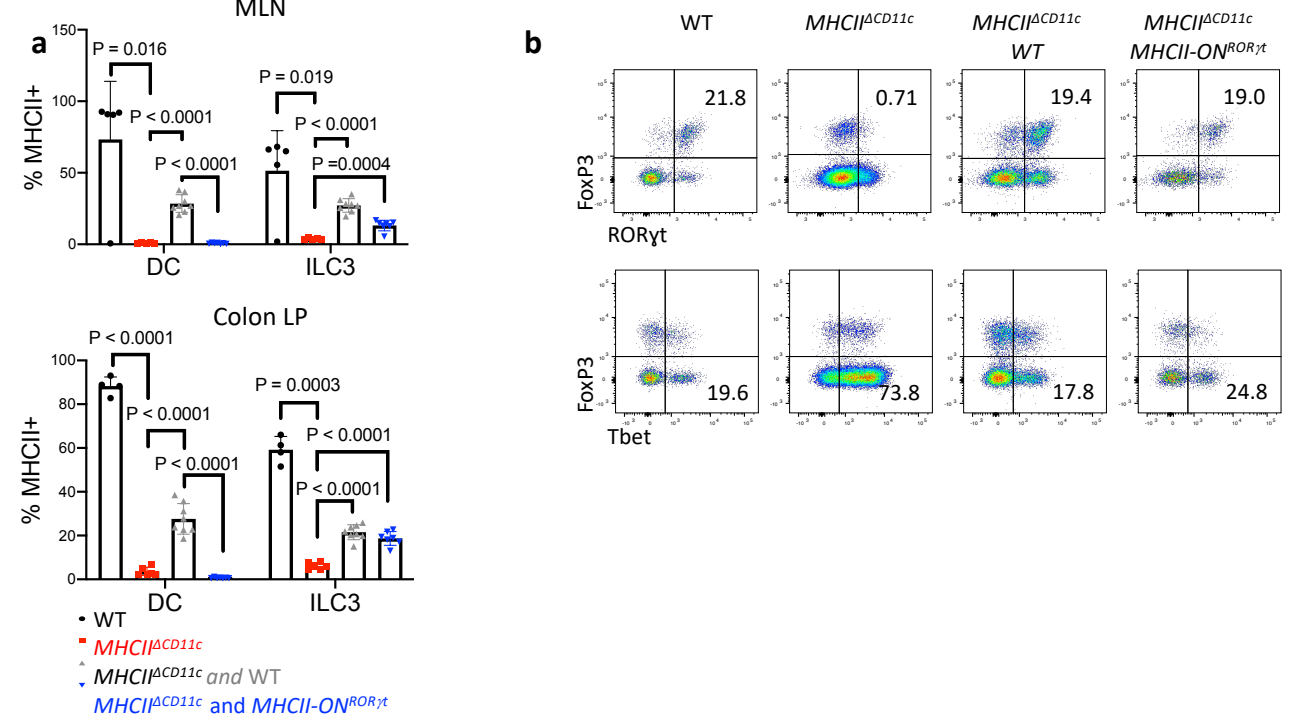

\section{Extended Data Fig. 7. Gain-of-function expression of MHCII in ILC3 rescues bone marrow-} derived iTreg cell differentiation. a, Aggregate data showing MHCll frequency on donor-derived DC and ILC3 in MLN and colon lamina propria from chimeric mice reconstituted with combinations of donor BM cells as indicated, with representative flow cytometry panel in Fig. 5b. b, Donor bone marrow-derived $\mathrm{CD}^{+} \mathrm{T}$ cells differentiation in colon lamina propria from chimeric mice reconstituted with combinations of BM cells as indicated. MLN: WT $(n=5), \operatorname{MHCII}^{\triangle C D 11 c}(n=5)$, $\mathrm{MHCII}^{\triangle C D 11 c}$ and WT (n=8), MHCII ${ }^{\triangle C D 11 c}$ and MHCII-ON ${ }^{\triangle R O R y t}(n=7)$. Colon: WT $(n=4), \mathrm{MHCI}^{\triangle C D 11 c}$ $(n=6), \mathrm{MHCII}^{\triangle C D 11 c}$ and WT $(n=8), \mathrm{MHCI}^{\triangle \mathrm{CD} 11 \mathrm{c}}$ and $\mathrm{MHCll}^{-O N^{\triangle R O R Y t}}(\mathrm{n}=7)$. All statistics were calculated by unpaired two-sided Welch's t-test. Error bars denote mean \pm s.d. $p$-values are indicated in the figure. 


\section{Methods}

\section{Mice}

Mice were bred and maintained in the Alexandria Center for the Life Sciences animal facility of the New York University School of Medicine, in specific pathogen-free conditions. C57BL/6 mice (Jax\# 000664), Batf3-/(B6.129S(C)-Batf3tm1Kmm/J \#Jax 013755), Itgav ${ }^{f / f}$ (B6.129P2(Cg)Itgav $^{\text {tm2Hyn } / J ~ J a x \# ~ 032297, ~ C D 45.1 ~ m i c e ~(B 6 . S J L-P t p r c a ~ P e p c b / B o y J, ~ J a x \# ~ 002014), ~ C D 4-C r e ~}$ (Tg(Cd4-cre)1Cwi/BfluJ, Jax\# 017336), CD11C-Cre (B6.Cg-Tg(Itgax-cre)1-1Reiz/J \#Jax 008068), $\operatorname{Ccr}^{-/}\left(B 6.129 P 2(C)-C c r 7 t m 1 R f o r / J\right.$, Jax\# 006621), I-AB ${ }^{f / f}$ (B6.129X1-H2-Ab1tm1Koni/J \#Jax 013181), Zbtb46-Cre (B6.Cg-Zbtb46tm3.1(cre)Mnz/J \#Jax 028538), Zbtb46-eGFP (B6.129S6(C)-Zbtb46tm1.1Kmm/J \#Jax 027618), tdTomato ${ }^{\text {LSL } \quad(B 6 ; 129 S 6-}$ Gt(ROSA)26Sortm14(CAG-tdTomato)Hze/J \#Jax 007908), Aire ${ }^{\text {f/f }}$ (B6.Cg-Airetm1Dfil/J \#Jax 031409), Nur77-eGFP (C57BL/6-Tg(Nr4a1-EGFP/cre)820Khog/J \#Jax 016617), CD90.1 (B6.PLThy1a/CyJ \#Jax 000406) mice were purchased from Jackson Laboratories. ROR $\gamma$ t-Cre and Hh7$2 \operatorname{tg}$ were generated in our laboratory and previously described ${ }^{3,38}$. BAC-transgenic Rorc(t)-GfpTG were generated by $\mathrm{G}$. Eberl's lab ${ }^{39}$. huLangerin (CD207)-DTA mice were kindky provided by Daniel H. Kaplan ${ }^{40}$. Ccr7 $7^{f / f}$ mice were previously described ${ }^{23}$. KKate $^{L S L}$ mice $^{41}$ were provided by Scott Lowe. I-AB lox-STOP-lox ${ }^{34}$, Aire-DTR ${ }^{42}$, and H2-DMa1 ${ }^{\text {f/f }}$ mice have been described ${ }^{21}$. Littermates with matched sex (both males and females) were used. Mice in all the experiments were 6-12 weeks old at the starting point of treatment. Animal sample size estimates were determined using power analysis (power $=90 \%$ and alpha $=0.05$ ) based on the mean and standard deviation from our previous studies and/or pilot studies using 4-5 animals per group. All animal procedures were performed in accordance with protocols approved by the Institutional Animal Care and Usage Committee of New York University School of Medicine. 


\section{Antibodies, intracellular staining and flow cytometry}

The following monoclonal antibodies were purchased from eBiosciences, BD Pharmingen or BioLegend: CD3 (145-2C11), CD4 (RM4-5), CD25 (PC61), CD44 (IM7), CD45.1 (A20), CD45.2 (104), CD90.1 (HIS51), CD90.2 (53-2.1), CD19 (1D3), CD45R (RA3-6B2), CD127 (A7R34), CD51 (RMV-7), IA/IE (56-5321-82), CCR6 (3D6), Ncr1 (29A1.4), NK1.1 (PK136), CD62L (MEL-14), CXCR5 (L138D7), TCRß (H57-597), TCR Vß6 (RR4-7), Bcl-6 (K112-91), Foxp3 (FJK-16s), RORyt (B2D or Q31-378), T-bet (eBio4B10), IL-17A (eBio17B7) and IFN-ץ (XM61.2), CD11C (N418), CD11b (M1/70), CX3CR1 (SA011F11), Ly6c (HK1.4), SIRPa (P84), Ly6G (1A8), CD273 (TY25), Clec12a (5D3), CD103 (M290), XCR1 (ZET), F4/80 (BM8), CCR7 (4B12), 4',6-diamidino2-phenylindole (DAPI) or Live/dead fixable blue (ThermoFisher) was used to exclude dead cells.

For transcription factor staining, cells were stained for surface markers, followed by fixation and permeabilization before nuclear factor staining according to the manufacturer's protocol (Foxp3 staining buffer set from eBioscience). For cytokine analysis, cells were incubated for $5 \mathrm{~h}$ in RPMI with $10 \%$ FBS, phorbol 12-myristate 13-acetate (PMA) $(50 \mathrm{ng} / \mathrm{ml}$; Sigma), ionomycin (500 ng/ml; Sigma) and GolgiStop (BD). Cells were stained for surface markers before fixation and permeabilization, and then subjected to intracellular cytokine staining according to the manufacturer's protocol (Cytofix/Cytoperm buffer set from BD Biosciences). Flow cytometric analysis was performed on an LSR II (BD Biosciences) or Cytek Aurora (Cytek) or an Aria II (BD Biosciences) and analyzed using FlowJo software (Tree Star).

\section{Gating Strategy}

Hh7-2 gating: FSC, SSC; Live Dead',singlets, Dump- (B220,TCRgd, Ly6G), MHCIl-, CD4+, $\mathrm{TCR}^{+}, \mathrm{VB}^{+}, \mathrm{CD} 45.1^{+}$or $\mathrm{CD}^{+} 0.1^{+}$; cDC gating: FSC, SSC; Live Dead ${ }^{-}$, singlets, Dump (B220,TCRgd, Ly6G), CD11c ${ }^{+}$and CD11 ${ }^{+}$, SIRPalow-moderate (remove CD11c- SIRPA $^{\text {high }}$ ); cDC2 gating: FSC, SSC; Live Dead',singlets, Dump- (B220,TCRgd, Ly6G), CD11 ${ }^{+}$and CD11 ${ }^{+}$, SIRPa ${ }^{\text {low-moderate }}$ (remove CD11c', SIRPA ${ }^{\text {high }}$ ), Clec12a- SIRPa ${ }^{+}$; migratory cDC2 gating: FSC, 
SSC; Live Dead',singlets, Dump ${ }^{-}$(B220,TCRgd, Ly6G), CD11c ${ }^{+}$and CD11 $\mathrm{b}^{+}$, SIRPalow-moderate

(remove CD11c-, SIRPA ${ }^{\text {high }}$ ), Clec12a- SIRPa ${ }^{+}, \mathrm{PDL}^{+}$; Lti-like ILC3 gating: FSC, SSC; Live Dead',singlets, Dump- (B220,TCRgd, Ly6G), TCR $\beta^{-}, \mathrm{CD}^{-} 0^{+}, \mathrm{II7R}{ }^{+}, \mathrm{ROR} \gamma \mathrm{t}^{+}, \mathrm{CCR} 6^{+}, \mathrm{CD}^{+} 5^{+}$; NCR1+ ILC3 gating: FSC, SSC; Live Dead',singlets, Dump' (B220,TCRgd, Ly6G), TCR $\beta^{-}$, $\mathrm{CD} 90+, \mathrm{II7R}+, \mathrm{ROR}_{\gamma t^{+}}, \mathrm{CCR}^{-}, \mathrm{NCR}^{+}$

\section{Isolation of lymphocytes and APCs}

After removal of caecal patches, large intestine tissues were sequentially treated with PBS containing $1 \mathrm{mM}$ DTT at room temperature for $10 \mathrm{~min}$, twice with $5 \mathrm{mM}$ EDTA at $37^{\circ} \mathrm{C}$ for $10 \mathrm{~min}$ to remove epithelial cells, and then minced and dissociated in digestion buffer (RPMI containing collagenase (1 mg ml-1 collagenase D; Roche), DNase I (100 $\mu \mathrm{g} \mathrm{ml-1;} \mathrm{Sigma),} \mathrm{dispase} \mathrm{(0.1} \mathrm{U}$ $\mathrm{ml}-1$; Worthington) and $10 \%$ FBS) with constant stirring at $37^{\circ} \mathrm{C} 55 \mathrm{~min}$. Leukocytes were collected at the interface of a $40 \% / 80 \%$ Percoll gradient (GE Healthcare). Lymph nodes were mechanically disrupted for lymphocyte isolation. For isolation of myeloid cells and ILC, lymph nodes were mechanically disrupted with digestion buffer with constant stirring at $37^{\circ} \mathrm{C} 30 \mathrm{~min}$.

\section{H. hepaticus culture and oral infection}

H. hepaticus was kindly provided by Dr. James Fox (MIT). Hh was cultured and administrated as was previously described ${ }^{3}$. Frozen stock aliquots of $H$. hepaticus were stored in Brucella broth with $20 \%$ glycerol and frozen at $-80^{\circ} \mathrm{C}$. The bacteria were grown on blood agar plates (TSA with $5 \%$ sheep blood, Thermo Fisher). Inoculated plates were placed into a hypoxia chamber (BillupsRothenberg), and anaerobic gas mixture consisting of $80 \%$ nitrogen, $10 \%$ hydrogen, and $10 \%$ carbon dioxide (Airgas) was added to create a micro-aerobic atmosphere, in which the oxygen concentration was $3 \sim 5 \%$. The micro-aerobic jars containing bacterial plates were left at $37^{\circ} \mathrm{C}$ for 4 days before animal inoculation. For oral infection, $H$. hepaticus was resuspended in Brucella 
broth by application of a pre-moistened sterile cotton swab applicator tip to the colony surface. $0.3 \mathrm{~mL}$ bacterial suspension was administered to each mouse by oral gavage. Mice were inoculated for a second dose after 3 days.

\section{Adoptive transfer of Hh7-2 TCR transgenic cells}

Adoptive transfer of Hh7-2 was done as was previously described ${ }^{3}$, with minor modifications. Recipient mice were colonized with $H$. hepaticus by oral gavage seven days before adoptive transfer. Spleens and lymph nodes from donor Hh7-2 TCRtg mice were collected and mechanically disassociated. Red blood cells were lysed using ACK lysis buffer (Lonza). Naive Hh7-2 T cells were sorted as CD4 ${ }^{+} \mathrm{TCR} \beta^{+} \mathrm{CD} 44^{\mathrm{l}} \mathrm{CD} 62 \mathrm{~L}^{\text {hi }} \mathrm{CD} 25^{-} \mathrm{V} \beta 6^{+}(\mathrm{HH} 7-2 \mathrm{tg})$, on the Aria II (BD Biosciences). For analysis of early differentiation, cells were additionally labeled with CFSE (ThermoFisher). Cells were resuspended in PBS on ice and $100 \mathrm{~K}$ were transferred into congenic isotype-labelled recipient mice by retro-orbital injection. Cells from MLN were analyzed 3 days after transfer and cells from colon LP were analyzed 10-14 days after transfer.

\section{CITE-seq}

CITE-seq and cell hashing were performed as described ${ }^{43,44}$ with minor modifications. Single-cell suspensions were obtained from digests of C1 MLN of tdTomato-ON ${ }^{C D 11 c}$ or tdTomato-ON ${ }^{R O R \gamma t}$; zbtb46-eGFP mice that had been colonized with Helicobacter for 7 days. Cells were sorted on a BD FACSAriall using a 100- $\mu \mathrm{m}$ nozzle. Dead cells as well as T cells and B cells were gated out using DAPI, TCR $\beta, T C R \gamma \delta$ and B220 antibodies. From tdTomato-ON ${ }^{C D 11 c}$ mice, the tdTomato+ population was collected separately from two mice. From $t d T o m a t o-O N^{R O R \gamma t} ;$ zbtb46-eGFP mice, we collected 3 populations from two separate mice: $\mathrm{GFP}^{+}, \mathrm{GFP}^{+}$tdTomato ${ }^{+}$and tdTomato ${ }^{+}$. Sorted cells were stained separately with hashing antibodies (Biolegend) ${ }^{44}$. After removal of excess hashing antibodies, we combined the samples and stained them with CITE-seq 
antibodies, conjugated using iEDDA click chemistry to barcode oligos as described before ${ }^{45}$. In addition, we included some commercially available Totalseq-A antibodies for CD11c, CD90.2, CD185, CD51 and CD127 (Biolegend). Post-sorting and staining, cells were run through the standard 10x Chromium (v3) protocol up until cDNA amplification, with the following modification: For the cDNA PCR step, 0.2uM of ADT additive primer (5'CCTTGGCACCCGAGAATTCC) and 0.2uM of HTO additive primer (5'GTGACTGGAGTTCAGACGTGTGCTC) were added to the cDNA amplification master mix. Post cDNA amplification, a 0.6X SPRI cleanup was performed to separate the cDNA fraction (on beads) from the smaller ADT and HTO fractions (in supernatant). The cDNA fraction was converted into a 3' tag gene expression library according to the $10 x$ Genomics Single Cell Genomics Protocol (v3). Supernatant from cleanup was kept for ADT and HTO preparation.

To the supernatant, another $1.4 \mathrm{X}$ SPRI was added to bring the total SPRI concentration to $2 \mathrm{X}$. After washing the beads in $80 \%$ ethanol and eluting in water, a second round of $2 X$ SPRI cleanup was performed to remove any residual primer carryover from the cDNA PCR. Post cleanup, eluate was taken into ADT PCR amplification (using TruSeq Small RNA RPIx primer (5'CAAGCAGAAGACGGCATACGAGXXXXXXXXGTGACTGGAGTTCCTTGGCACCCGAGAAT TCCA) and SI PCR primer

(5'AATGATACGGCGACCACCGAGATCTACACTCTTTCCCTACACGACGCTC)), and into hashtag amplification (using TruSeq D7xx

(5'CAAGCAGAAGACGGCATACGAGATXXXXXXXXGTGACTGGAGTTCAGACGTGTGC) and SI PCR primers). Libraries were pooled and sequenced on a 100 cycle Novaseq S1 flowcell, with the configuration of 30 base pairs for R1, and 92 base pairs for R2. Additional protocol details can be found for CITE-seq and cell hashing at www.cite-seq.com. 
Post sequencing, gene expression count matrices were generated using cellranger version 5.0 using the refdata-gex-mm10-2020-A reference library provided by $10 x$ Genomics, with the additional sequences of cre, eGFP, and tdTomato. Counts matrices for hashtags and antibodies were generated using CITE-seq-Count version 1.4.4. Downstream analysis was then performed in $\mathrm{R}$ using Seurat.

Quality control and doublet removal: We initially selected all cells that were detected in our RNAseq, cell hashing, and ADT libraries. We removed cells with $<700$ detected genes, but also removed cells which had an aberrantly high number of genes (more than 5,000 genes) and a high percentage of mitochondria genes (more than 6\%). Additionally, we removed the cells which were attached by the clumps of antibodies and had a too high number of ADT or HTO UMIs (more than 5,000 ADT UMI and 4,000 HTO UMI). We used our previously described hashing-based doublet detection strategy ${ }^{44}$, implemented in HTODemux, to identify doublets that represent two or more cells representing different samples. Only Singlet cells were used for the downstream analysis.

Multimodal analysis: We normalized RNA data using SCTransform ${ }^{46}$ and applied the centeredlog ratio (CLR) transformation to normalize ADT data within each cell. We used principal component analysis (PCA) to reduce dimensionality of both datasets. Then we took both top 20 RNA and protein PCA dimensions as the input of weighted nearest neighbors (WNN) method ${ }^{18}$ to construct the multimodal weighted KNN graph. To cluster our multimodal dataset, we first used the weighted KNN graph to generate a shared nearest neighbor graph (SNN) and then apply the graph-based smart local moving (SLM) algorithm (https://doi.org/10.1140/epjb/e2013-40829-0) on this SNN graph to find clusters with 0.8 resolution. We performed differential expression on all pairs of clusters for both RNA and protein markers, and merged clusters that did not exhibit clear 
evidence of separation. All samples were clustered together and separated later for further analysis as indicated.

\section{CITE-seq data projection in Flowjo}

For gating analysis, scaled and normalized ADT counts together with cluster identity, UMAP and UWnn coordinates were exported into a csv format that was uploaded in FlowJo.

\section{RNAscope}

Fresh-frozen spleen and lymph nodes were sectioned at $8 u m$ and then fixed overnight at $4^{\circ} \mathrm{C}$ in $10 \%$ neutral buffered formalin (Thermo Fisher Scientific, Waltham, MA) before proceeding with an RNAscope RED 2.5 HD Chromogenic Assay kit (Advanced Cell Diagnostics, Newark, CA) for detection of Aire mRNA. DapB probe was used as negative control and Polr2a probe was used as positive control. Semi-quantitative scores were determined in a blinded fashion based on the number of Aire ${ }^{+}$cells per section.

\section{Integrin $\alpha_{v} \beta_{8}$ (ADWA-11) blocking Ab}

We injected IP 200ug of ADWA-11 into mice colonized with Hh for 7 days. On the same day we adoptively transferred 100K CFSE-labeled naive Hh7-2 cells and tested their proliferation and differentiation in the C1 MLN, 3 days after the transfer.

\section{Generation of bone marrow (BM) chimeric reconstituted mice}

To generate chimeric mice, 4-5 week old CD45.1 mice were irradiated twice with 500 rads/mouse at an interval of 2-5 h (X-RAD 320 X-Ray Irradiator). A day after, bone marrow (BM) mononuclear cells were isolated from donor mice, as indicated in each experiment, by flushing the femur bones. Red blood cells were lysed with ACK Lysing Buffer, and lymphocytes were depleted for Thy1.2 
using magnetic microbeads (Miltenyi). BM cells were resuspended in PBS and a total 3-4 x $10^{6}$ BM cells were injected intravenously into the irradiated mice. In case of mixed BMC reconstitution, a ratio of 1:1 was used. Mice were kept for a week on broad spectrum antibiotics $(1 \mathrm{mg} / \mathrm{mL}$ sulfamethoxazole and $0.2 \mathrm{mg} / \mathrm{mL}$ trimethoprim), followed by microbiome reconstitution by fecal gavage. Mice were reconstituted for 1-2 months before Hh colonization. After 7 weeks, peripheral blood samples were collected and analyzed by FACS 7 to check for reconstitution.

\section{Intravital multiphoton microscopy}

Naive Hh7-2 T cells were isolated from Nur77-eGFP Hh7-2tg mice, labeled with Cell tracker dye (eBioscience Cell Proliferation Dye eFluor 450), and transferred into $m K a t e 2-O N^{z b t b 46} ; R O R \gamma t-$ eGFP mice that had been colonized with Hh for 6 days. Fifteen hours following adoptive transfer of $\mathrm{Hh}$ T cells, mice were euthanized and C1 MLN were immediately isolated and mounted in cold RPMI with $10 \%$ FCS for intravital multiphoton microscopy. Image stacks were acquired with an Olympus multiphoton FVMPE-RS system equipped with both InSight X3 and Mai Tai Deepsee (Spectra-Physics) tunable Ti:Sapphire lasers. To acquire serial optical sections, a laser beam (780 nm for eFluor ${ }^{\text {TM }} 450$ and $940 \mathrm{~nm}$ for simultaneous excitation of eGFP and mKate2) was focused through a water immersion lens (N.A. 1.05; Olympus) and scanned with a field of view of $0.5 \mathrm{~mm}^{2}$, at $600 \mathrm{~Hz}$. Z-stacks were acquired in $2 \mathrm{~mm}$ steps to image a total depth of $150-200 \mathrm{~mm}$ of tissue.

\section{Image analysis}

Raw image stacks were imported into Fiji (NIH) for T cell colocalization analysis. Provided images are presented as a maximal projection of 3-6 $\mathrm{mm}$ optical sections. For visualizing individual labelled cells expressing both the mKate2 and eGFP, the brightness and contrast were adjusted accordingly to single positive green (eGFP) and red (mKate2) cells. Adoptively transferred Hh7- 
$2 \operatorname{tg}$ T cells were identified via positive labeling with cell proliferation dye eFluor 450. Primed Hh7$2 \operatorname{tg}$ T cells were identified via expression of the Nur77-eGFP reporter. Cell identity was scored by a combination of both fluorescent reporter expression as well as cell morphology. Specifically, cells expressing mKate2 with a dendritic cell shape were scored as $\mathrm{cDC}$, while cells expressing both RORyt-eGFP and mKate2 (or eGFP alone) with an amoeboid (non-spherical) cell shape were scored as ILC3. T cell interactions with CDC or ILC3 was strictly measured as direct $(<1$ micron) colocalization of cells with respective fluorescent and cell morphology combinations.

\section{Statistical analysis}

For animal studies, mutant and control groups did not always have similar standard deviations and therefore an unpaired two-sided Welch's t-test was used. Error bars represent \pm s.d. Animal sample size estimates were determined using power analysis (power $=90 \%$ and $\alpha=0.05$ ) based on the mean and s.d. from our previous studies and/or pilot studies using 4-5 mice. No samples were excluded from analysis.

\section{Additional References}

38 Eberl, G. \& Littman, D. R. Thymic origin of intestinal alphabeta T cells revealed by fate mapping of RORgammat+ cells. Science 305, 248-251, doi:10.1126/science.1096472 (2004).

39 Lochner, M. et al. In vivo equilibrium of proinflammatory IL-17+ and regulatory IL-10+ Foxp3+ RORgamma $\mathrm{t}+\mathrm{T}$ cells. The Journal of experimental medicine 205, 1381-1393, doi:10.1084/jem.20080034 (2008).

40 Kaplan, D. H., Jenison, M. C., Saeland, S., Shlomchik, W. D. \& Shlomchik, M. J. Epidermal langerhans cell-deficient mice develop enhanced contact hypersensitivity. Immunity 23, 611620, doi:10.1016/j.immuni.2005.10.008 (2005). 
41 Dow, L. E. et al. Conditional reverse tet-transactivator mouse strains for the efficient induction of TRE-regulated transgenes in mice. PLoS One 9, e95236, doi:10.1371/journal.pone.0095236 (2014).

42 Metzger, T. C. et al. Lineage tracing and cell ablation identify a post-Aire-expressing thymic epithelial cell population. Cell Rep 5, 166-179, doi:10.1016/j.celrep.2013.08.038 (2013).

43 Stoeckius, M. et al. Simultaneous epitope and transcriptome measurement in single cells. Nat Methods 14, 865-868, doi:10.1038/nmeth.4380 (2017).

44 Stoeckius, M. et al. Cell Hashing with barcoded antibodies enables multiplexing and doublet detection for single cell genomics. Genome Biol 19, 224, doi:10.1186/s13059-018-1603-1 (2018).

45 van Buggenum, J. A. et al. A covalent and cleavable antibody-DNA conjugation strategy for sensitive protein detection via immuno-PCR. Sci Rep 6, 22675, doi:10.1038/srep22675 (2016).

46 Hafemeister, C. \& Satija, R. Normalization and variance stabilization of single-cell RNA-seq data using regularized negative binomial regression. Genome Biol 20, 296, doi:10.1186/s13059-019-1874-1 (2019). 